\title{
TWO NEW CONDITIONS SUPPORTING THE FIRST-ORDER APPROACH TO MULTI-SIGNAL PRINCIPAL-AGENT PROBLEMS
}

\author{
BY JOHN R. CONLON ${ }^{1}$
}

July 16, 2008

\begin{abstract}
This paper presents simple new multi-signal generalizations of the two classic methods used to justify the first-order approach to moral hazard principal-agent problems, and compares these two approaches with each other. The paper first discusses limitations of previous generalizations. It then uses a state-space formulation to obtain a new multi-signal generalization of the Jewitt (1988) conditions. Next, using the Mirrlees formulation, the paper obtains new multi-signal generalizations of the CDFC approach of Rogerson (1985) and Sinclair-Desgagné (1994). Vector calculus methods are used to derive easy-to-check local conditions for our generalization of the CDFC. Finally, we argue that the Jewitt conditions may generalize more flexibly than the CDFC to the multi-signal case. This is because, with many signals, the principal can become very well informed about the agent's action and, even in the one-signal case, the CDFC must fail when the signal becomes very accurate.
\end{abstract}

\section{KEYWORDS}

Principal-Agent Model, Moral Hazard, First-Order Approach, Multiple Signals

${ }^{1}$ The paper benefitted greatly from a faculty seminar at the University of Mississippi. Discussions with Richard Boylan, Gerard Buskes, Ron Harstad, David Marshall, Frank Page, Hyun Park, Paul Pecorino, Marcin Pȩski, William Rogerson, Bernard Sinclair-Desgagné, Jeroen Swinkels, and Min-hung Tsay were also extremely helpful. In particular, especially perceptive questions by Bernard Sinclair-Desgagné led to the results in Section 8, and contributed significantly to Section 10. Ian Jewitt also suggested numerous major improvements, only a few of which are explicitly acknowledged below. Finally, extensive and thoughtful suggestions by the referees and by the coeditor, Larry Samuelson, have greatly streamlined and improved the paper. This work was made possible, in part, by a sabbatical from the University of Mississippi in the Fall of 2005. All remaining errors are mine. 


\section{INTRODUCTION}

MORAL HAZARD PRINCIPAL-AGENT PROBLEMS have always presented surprising technical challenges. In particular, a great deal of attention has focused on determining conditions under which the principal can predict the agent's behavior using the agent's first order conditions alone. This question was first raised by Mirrlees (1999, originally circulated in 1975), and Guesnerie and Laffont (1978), and was further discussed in Grossman and Hart (1983). Rogerson (1985) gave a rigorous set of sufficient conditions for the first-order approach in the one-signal case, based on the "Convexity of the Distribution Function Condition" (CDFC). Jewitt (1988) gave a different set of sufficient conditions, not using the CDFC, also for the one-signal case.

In addition, Jewitt (1988) gave two sets of sufficient conditions for the multi-signal case, one of which also avoided the CDFC. However, both sets of Jewitt's multi-signal conditions assumed that signals were independent of one another.

Six years later, Sinclair-Desgagné (1994) developed a generalization of the CDFC, the GCDFC, for the multi-signal case. This allowed him to avoid Jewitt's independence assumption. However, the GCDFC requires the marginal distribution on all but one of the principal's signals to be an affine (i.e., constant sloped) function of the agent's action (see Section 3). Also, a second less essential condition of his makes the marginal distribution on all but one of the principal's signals independent of the agent's action.

In addition, the CDFC and GCDFC do not capture the standard diminishing marginal returns intuition. As Rogerson (1985) explains, "if output is determined by a stochastic production function with diminishing returns to scale in each state of nature, the implied distribution function over output will not, in general, exhibit the CDFC." Thus, neither Rogerson's one-signal results nor Sinclair-Desgagné's multi-signal results build on the usual economic notion of diminishing marginal returns. 
Jewitt's (1988) conditions, by contrast, do build on the standard economic sense of diminishing returns, so his conditions are closer to ordinary economic intuition. However, the profession seems to have regarded his conditions as overly technical. Thus, while most principal-agent textbooks present the Rogerson CDFC, none present the Jewitt conditions. Also, little theoretical work has built on these conditions. In particular, no multi-signal generalizations of Jewitt's conditions are available, beyond those in Jewitt's original paper, which assume independent signals.

The goal of this paper is to clarify and extend both of the above methods of justifying the first-order approach. First we suggest that the Rogerson conditions arise most naturally from the Mirrlees formulation of the agent's problem, while the Jewitt conditions arise naturally from the older state-space perspective of Spence and Zeckhauser (1971) and Ross (1973). In addition we discuss limitations of previous multi-signal generalizations of the Jewitt and Rogerson one-signal approaches.

We next present new multi-signal extensions of each set of conditions, which are more general than previous extensions. The multi-signal generalization of Jewitt's approach yields essentially Jewitt's main set of multi-signal conditions, but without his restrictive independence assumption. The multi-signal extension of the CDFC approach involves a new condition, the Concave Increasing-Set Probability (CISP) condition, which generalizes the CDFC, and is more flexible than previous extensions. In addition, if we want to allow for a risk averse principal, we need an additional condition, the Nondecreasing Increasing-Set Probability (NISP) condition.

Finally, we argue that the Jewitt approach may generalize more flexibly to the multi-signal case than the CDFC approach. This is because, with many signals, the principal tends to become very well informed about the agent's action and, even in the one signal case, the CDFC must fail when the signal becomes very accurate. 
The multi-signal case is of practical interest since, in most actual environments, the principal sees a wide range of signals of an agent's effort. The multi-signal case is also especially interesting because it is often used to underscore the central role of information in principal-agent problems (Holmström (1979), Shavell (1979)).

The next section introduces the basic framework. Section 3 discusses previous results, and Section 4 presents the new multi-signal generalization of Jewitt's conditions. Sections 5 and 6 derive simple new multi-signal generalizations of the CDFC approach, for risk neutral and risk averse principals, respectively. Section 7 reduces the key NISP and CISP conditions to the existence of probability density flows having certain local properties. Section 8 shows how to construct these density flows for a given distribution function, and so, check NISP and CISP. Section 9 briefly discusses other extensions. Section 10 compares the two main approaches and concludes. ${ }^{2}$

\section{BASIC FRAMEWORK}

This section presents the general framework for multi-signal principal-agent problems, describing both the state-space and the Mirrlees formulations of the problem. Assume one principal and one agent. The agent chooses an effort level $a \geq 0$. This is combined with a random vector of state variables $\tilde{\vartheta}$, with probability density function $p(\vartheta)$, to generate a random vector of signals,

$$
\tilde{\mathbf{x}}=\mathbf{x}(a, \tilde{\vartheta})
$$

with $\mathbf{x}(\cdot, \cdot)$ taking values in $\mathbf{R}^{n}$. Equation $\left(1_{\mathrm{SS}}\right)$ is a state-space representation of the technology.

2 This paper is mostly self contained. The main exceptions are that (a) I have not derived the principal's standard first order conditions, (7) and (15) below, and (b) I have not included Jewitt's (1988) argument that $\mu \geq 0$ in (7). 
Assume, until Section 6, that the principal is risk neutral. The agent has a von Neumann-Morgenstern utility function $u(s)-a$, where $s$ is the agent's monetary payoff, and $u(\cdot)$ is a strictly increasing function. ${ }^{3}$ Let $s=s(\mathbf{x})$ be the function, chosen by the principal, specifying her payment to the agent as a function of the signal $\mathbf{x}$. Let the value of output be given by the function $\pi(\mathbf{x})$, as in Sinclair-Desgagné (1994). Then the principal's expected payoff is

$$
V(s(\cdot), a)=\int[\pi(\mathbf{x}(a, \vartheta))-s(\mathbf{x}(a, \vartheta))] p(\vartheta) d \vartheta
$$

and the agent's expected payoff is

$$
U(s(\cdot), a)=\int u(s(\mathbf{x}(a, \vartheta))) p(\vartheta) d \vartheta-a .
$$

The principal's problem is then to choose a payment schedule, $s^{*}(\cdot)$, and target action, $a^{*}$, by the agent, to maximize $\left(2_{\mathrm{SS}}\right)$, given two constraints:

$$
a^{*} \text { maximizes the expected payoff, } U\left(s^{*}(\cdot), a\right) \text {, to the agent, }
$$

and

$$
\text { the resulting expected payoff to the agent, } U\left(s^{*}(\cdot), a^{*}\right) \geq U_{0} \text {, }
$$

where $U_{0}$ is the agent's reservation utility. Here (4) and (5) are the usual incentive compatability and participation (individual rationality) constraints.

3 This function could easily be replaced by $u(s)-c(a)$ with $c(\cdot)$ convex. However, the curvature of $c(\cdot)$ can be important. For if we let $\hat{a}=c(a)$, then utility becomes $u(s)-\hat{a}$, but $\left(1_{\mathrm{SS}}\right)$ becomes $\tilde{\mathbf{x}}=\mathbf{x}\left(c^{-1}(\hat{a}), \tilde{\vartheta}\right)$. Thus, if $c(\cdot)$ is concave then $c^{-1}(\cdot)$ is convex, which can alter the production technology in ways that overturn some of the conditions below. See Brown et al. (1986) on the importance of the curvature of $c(a)$. 
To rephrase this in terms of a Mirrlees representation, let

$$
F(\mathbf{x} \mid a)=\operatorname{Prob}(\mathbf{x}(a, \tilde{\vartheta}) \leq \mathbf{x})
$$

be the cumulative distribution function of $\tilde{\mathbf{x}}$ implied by $\left(1_{\mathrm{SS}}\right)$ (where $\mathbf{x} \leq \mathbf{y}$, for $\mathbf{x}$, $\mathbf{y} \in \mathbf{R}^{n}$ means $\left.x_{i} \leq y_{i}, i=1,2, \ldots, n\right)$, and let $f(\mathbf{x} \mid a)$ be the corresponding density function. Assume as usual that the support of $f(\mathbf{x} \mid a)$ is compact and independent of $a$, and that $f(\mathbf{x} \mid a)$ is sufficiently smooth and bounded between two positive constants on its support. Using the Mirrlees notation, the principal's expected payoff becomes

$$
V(s(\cdot), a)=\int[\pi(\mathbf{x})-s(\mathbf{x})] f(\mathbf{x} \mid a) d \mathbf{x},
$$

and the agent's expected payoff becomes

$$
U(s(\cdot), a)=\int u(s(\mathbf{x})) f(\mathbf{x} \mid a) d \mathbf{x}-a .
$$

The principal's problem is still to maximize $V(s(\cdot), a)$ subject to (4) and (5).

Our primary focus is $\left(3_{\mathrm{SS}}\right)$ and $\left(3_{\mathrm{M}}\right)$. These are equivalent ways of representing the agent's expected payoff in terms of the payment function $s(\cdot)$ and the agent's action $a$. However, $\left(3_{\mathrm{SS}}\right)$ explicitly separates out the production technology $\mathbf{x}(a, \vartheta)$ from the density $p(\vartheta)$, while $\left(3_{\mathrm{M}}\right)$ folds them together in $f(\mathbf{x} \mid a)$. On the other hand, the state space representation contains extraneous information since many different state space representations correspond to any given Mirrlees representation (see Section 4).

The first-order approach assumes that one can replace the incentive compatibility constraint (4) by a "relaxed" constraint - i.e., the agent's first order condition

$$
U_{a}\left(s^{*}(\cdot), a^{*}\right)=0,
$$

where subscripts denote partial derivatives. To ensure that (6) implies (4), it is sufficient for the agent's utility, $U\left(s^{*}(\cdot), a\right)$, to be a concave function of her effort $a$. The goal of the first-order approach is to find conditions which yield this concavity. 
If one does replace (4) by (6) in the principal's problem, then the principal's optimal contract, $s^{*}(\mathbf{x})$, for inducing the agent to choose some action $a^{*}$ at minimum cost, satisfies the principal's usual first order condition

$$
\frac{1}{u^{\prime}\left(s^{*}(\mathbf{x})\right)}=\lambda+\mu \frac{f_{a}\left(\mathbf{x} \mid a^{*}\right)}{f\left(\mathbf{x} \mid a^{*}\right)},
$$

where $\lambda$ and $\mu$ are the Lagrange multipliers for the participation and relaxed incentive compatibility constraints, (5) and (6), respectively (see Jewitt, Kadan and Swinkels (2008), for an elegant treatment). In addition, as shown by Jewitt (1988, p. 1180), if (6) and (7) hold, then $\mu \geq 0$. Jewitt's argument also applies to the multi-signal case (Jewitt, p. 1184).

With this setup, conditions supporting the first order approach are generally derived using the following clever argument. Assume that $s^{*}(\cdot)$ solves the principal's relaxed problem, maximizing $V(s(\cdot), a)$, subject to (5) and (6). Thus $s^{*}(\cdot)$ satisfies (7). Suppose also that, if (7) holds, then one can use this to show that $U\left(s^{*}(\cdot), a\right)$ is concave in $a$. Then this same $s^{*}(\cdot)$ also solves the principal's original problem, maximizing $V(s(\cdot), a)$ subject to the more restrictive unrelaxed constraints (4) and (5). Any solution to the relaxed problem is therefore also an optimal solution to the principal's original unrelaxed problem, so the first order approach is justified.

\section{PREVIOUS RESULTS}

As indicated above, the first-order approach looks for conditions to ensure that the agent's utility, $U\left(s^{*}(\cdot), a\right)$, is a concave function of her effort $a$, if $s^{*}(\cdot)$ satisfies $(7)$. Rogerson (1985) and Jewitt (1988) obtain conditions which ensure this concavity in the one-signal case. Jewitt also presents some multi-signal generalizations of a slightly more restrictive version of his one-signal conditions. Sinclair-Desgagné (1994) obtains a multi-signal generalization of the Rogerson conditions. 
Whether we obtain the Rogerson/Sinclair-Desgagné conditions or the Jewitt conditions depends on whether we represent $U\left(s^{*}(\cdot), a\right)$ using $\left(3_{\mathrm{M}}\right)$ or $\left(3_{\mathrm{SS}}\right)$. If we represent $U\left(s^{*}(\cdot), a\right)$ using $\left(3_{\mathrm{M}}\right)$, (i.e., $\left.\int u\left(s^{*}(\mathbf{x})\right) f(\mathbf{x} \mid a) d \mathbf{x}-a\right)$, we are naturally led to impose conditions on $f(\mathbf{x} \mid a)$, since that is where $a$ appears. This yields the approach of Rogerson (1985) and Sinclair-Desgagné (1994), as is well known.

On the other hand, if we represent $U\left(s^{*}(\cdot), a\right)$ using the state-space formulation, $\left(3_{\mathrm{SS}}\right)$ (i.e., $\left.\int u\left(s^{*}(\mathbf{x}(a, \vartheta))\right) p(\vartheta) d \vartheta-a\right)$, then it becomes obvious that $U\left(s^{*}(\cdot)\right.$, $\left.a\right)$ is concave in $a$ if $\mathbf{x}(a, \vartheta)$ is concave in $a, s^{*}(\mathbf{x})$ is nondecreasing concave in $\mathbf{x}$, and $u(s)$ is nondecreasing concave in $s$. This leads naturally to the main set of Jewitt's (1988) multi-signal conditions. Note that Jewitt himself also used both the state space and Mirrlees formulations in his derivations.

However, both the Jewitt and the Sinclair-Desgagné multi-signal generalizations remain more restrictive than necessary. First, Jewitt's multi-signal results assume that the signals are independent from one another, though his proof of his main result does not require this independence.

Nevertheless, the Mirrlees formulation, which Jewitt uses to present his results, makes it difficult to even state his multi-signal results without assuming independence. Section 4 below therefore initially uses the state-space formulation to state and prove our generalization of Jewitt's main multi-signal results. A second major goal of that section is then to show how to restate these results using the Mirrlees formulation.

Sinclair-Desgagné's generalization of the Rogerson conditions is also more restrictive than necessary. As we show next, one of Sinclair-Desgagné's conditions, his Assumption (A8), requires the marginal distribution of all but one of the signals to be constant in $a$, and so, independent of the agent's action. That is, it requires all but one of the signals to be "ancillary" to the agent's action (Lehmann (1983, pp. 45-46)). 
This assumption can be avoided if the principal is risk neutral. ${ }^{4}$ However, a second key assumption, his Assumption (A9), cannot be so easily avoided. Also, Assumption (A9) implies that the marginal distribution of all but one of the signals is an affine (i.e., constant sloped) function of the agent's choice variable, $a$ (see below).

To obtain these results, translate Sinclair-Desgagné's key assumptions into the continuous signal-space case of the current paper. For any index $h=1,2, \ldots, n$, let $\mathbf{x}_{-h}=\left(x_{1}, x_{2}, \ldots, x_{h-1}, x_{h+1}, \ldots, x_{n}\right)$, and let

$$
Q\left(x_{h}^{0}, \mathbf{x}_{-h} \mid a\right)=\int_{x_{h}^{0}}^{\infty} f\left(x_{h}, \mathbf{x}_{-h} \mid a\right) d x_{h} .
$$

Intuitively $Q\left(x_{h}^{0}, \mathbf{x}_{-h} \mid a\right)$ is the probability density that the random point $\tilde{\mathbf{x}}$ is on the upper ray, starting from $\left(x_{h}^{0}, \mathbf{x}_{-h}\right)$, pointing in the direction of increasing $x_{h}$. Thus, $Q\left(x_{h}^{0}, \mathbf{x}_{-h} \mid a\right)$ is a generalization of the upper cumulative distribution function to the multi-signal case, where this upper cumulative focuses on a one-dimensional ray in the $n$-dimensional space of signals. With this notation, Assumptions (A8) and (A9) are:

Assumption (A8) (Generalized Stochastic Dominance): For at least one index $h$, $Q\left(x_{h}^{0}, \mathbf{x}_{-h} \mid a\right)$ is nondecreasing in a for all $x_{h}^{0}$ and $\mathbf{x}_{-h}$.

Assumption (A9) (Generalized Concave (Upper) Distribution Function Condition): For at least one index $h, Q\left(x_{h}^{0}, \mathbf{x}_{-h} \mid a\right)$ is concave in a for all $x_{h}^{0}$ and $\mathbf{x}_{-h}$.

Proposition 1: If Assumption (A8) holds, then $f_{-h}\left(\mathbf{x}_{-h} \mid a\right)$, the marginal density of $\tilde{\mathbf{x}}$ on $\mathbf{x}_{-h}$, is independent of a for almost all $\mathbf{x}_{-h}$. If instead Assumption (A9)

4 This can be seen as follows. First, Sinclair-Desgagné's other key assumption, his Assumption (A9) (which we are about to discuss), implies the CISP condition of Section 5 below. Thus, by Proposition 4 in that section, (A9), plus the Monotone Likelihood Ratio property and $u(\cdot)$ strictly concave, justify the first order approach.

By contrast, if the principal is risk averse, then Proposition 5 in Section 6 is needed. This therefore requires the additional assumption, NISP, introduced below. SinclairDesgagné's Assumption (A8) plays the same role as NISP, but is more restrictive. 
holds, then $f_{-h}\left(\mathbf{x}_{-h} \mid a\right)$ is an affine function of a for almost all $\mathbf{x}_{-h}$, so $f_{-h}\left(\mathbf{x}_{-h} \mid a\right)=$ $\eta_{1}\left(\mathbf{x}_{-h}\right)+\eta_{2}\left(\mathbf{x}_{-h}\right)$ a, for some functions $\eta_{1}(\cdot)$ and $\eta_{2}(\cdot){ }^{5}$

Proof: Assume (A8). Then $Q\left(x_{h}^{0}, \mathbf{x}_{-h} \mid a\right)$ is nondecreasing in $a$ for all $x_{h}^{0}$ and $\mathbf{x}_{-h}$. Thus its limit as $x_{h}^{0} \rightarrow-\infty$ is also nondecreasing in $a$ for all $\mathbf{x}_{-h}$, so

$$
f_{-h}\left(\mathbf{x}_{-h} \mid a\right)=Q\left(-\infty, \mathbf{x}_{-h} \mid a\right)
$$

is nondecreasing in $a$ for all $\mathbf{x}_{-h}$. However, $\int f_{-h}\left(\mathbf{x}_{-h} \mid a\right) d \mathbf{x}_{-h}=1$. Thus, $f_{-h}\left(\mathbf{x}_{-h} \mid a\right)$ is constant in $a$ for almost all $\mathbf{x}_{-h}$.

In the same way, if Assumption (A9) holds, then (8) is concave in $a$ for all $\mathbf{x}_{-h}$. Thus, again using $\int f_{-h}\left(\mathbf{x}_{-h} \mid a\right) d \mathbf{x}_{-h}=1$, it now follows that $f_{-h}\left(\mathbf{x}_{-h} \mid a\right)$ is an affine function of $a$ for almost all $\mathbf{x}_{-h}$.

Q.E.D.

Note that this proposition does not imply that the $\tilde{\mathbf{x}}_{-h}$ signals are useless when (A8) holds, as Sinclair-Desgagné (2008) points out. For example, when a principal uses the output of other agents as a benchmark in "yardstick competition" (Holmström (1982), Mookherjee (1984)), this involves signals which are ancillary to the agent's action, but may nevertheless be quite important.

As a second example, suppose the principal uses a scale to weigh output. If the principal does not know how accurate the scale is, she may first put an object of known weight on the scale to calibrate it, before weighing the agent's output. In this case, the calibrating measurement is ancillary, but is nevertheless clearly relevant to the optimal payment schedule. It may therefore figure prominently in an optimal contract.

${ }^{5}$ Note that these conditions are necessary but not sufficient. In addition, even though $f_{-h}\left(\mathbf{x}_{-h} \mid a\right)$ constant in $a$ implies $f_{-h}\left(\mathbf{x}_{-h} \mid a\right)$ affine in $a$, it does not follow that (A8) implies (A9). Thus, suppose $\tilde{\mathbf{x}}_{-h}$ is ancillary, while the conditional cumulative distribution function, $F_{h}\left(x_{h} \mid \mathbf{x}_{-h}, a\right)$, is decreasing, but not convex, in $a$. Then $\tilde{\mathbf{x}}$ satisfies (A8) but not (A9). 
Multiple calibrating signals are also possible. One may then ask, e.g., how the optimal contract changes as the calibration becomes more and more precise.

Thus, although Assumption (A8) is restrictive, it does allow the $\tilde{\mathbf{x}}_{-h}$ signals to play an important role in helping the principal to interpret $\tilde{x}_{h}$. Alternatively, once the principal sees $\tilde{x}_{h}$, the $\tilde{\mathbf{x}}_{-h}$ signals may then contain a great deal of additional information. Similarly, if Assumption (A9) holds, it may nevertheless be the case that the distribution of $\tilde{\mathbf{x}}_{-h}$ given $\tilde{x}_{h}$ depends on $a$ in a highly nonlinear manner.

A related way to see this is that the contract ultimately depends on the likelihood ratio and, as Sinclair-Desgagné (2008) shows, this likelihood ratio can depend, in interesting ways, even on ancillary signals. Thus, Assumptions (A8) and (A9) do permit a wide range of interesting contracts. Nevertheless, Proposition 1 shows that these assumptions are fairly restrictive. The following sections therefore derive less restrictive versions of both the Jewitt and the Sinclair-Desgagné results.

\section{A NEW MULTI-SIGNAL EXTENSION OF JEWITT'S CONDITIONS}

This section uses the state-space formulation to give a simple generalization of Jewitt's (1988) main set of multi-signal conditions (for a brief discussion of his slightly different one-signal conditions, see footnote 7 below).

First, it is obvious from $\left(3_{\mathrm{SS}}\right)$ that concavity of $U\left(s^{*}(\cdot), a\right)$ in $a$ follows from two standard conditions plus one highly nonstandard condition:

(i) the coordinates of $\mathbf{x}(a, \vartheta)$ should be concave in $a$,

(ii) $s^{*}(\mathbf{x})$ should be nondecreasing concave in $\mathbf{x}$, and

(iii) $u(s)$ should be nondecreasing concave in $s$.

Conditions (i) and (iii) are completely standard: (i) is diminishing marginal productivity - at least when the signal is output - and (iii) is diminishing marginal utility, 
i.e., risk aversion. Condition (ii), however, is problematic, since it depends on the endogenous payment schedule, $s^{*}(\cdot)$. The condition therefore depends on the solution. Grossman and Hart's (1983) Proposition 9, however, gives conditions - in the onesignal case - which make the payment function $s^{*}(\mathbf{x})$ nondecreasing concave in $\mathbf{x}$, so (ii) is met. Moreover, these conditions generalize easily to the multi-signal case. Using the Mirrlees formulation, the principal's cost minimizing schedule, $s^{*}(\cdot)$, for inducing the agent to choose $a^{*}$, given the participation and relaxed incentive compatibility constraints (5) and (6), solves (7) above. Also, if (6) and (7) hold, then $\mu \geq 0$, as mentioned in the discussion after (7). In addition, even though the principal's relaxed constraint, (6), does not include the agent's complementary slackness condition at $a=0,(7)$ nevertheless holds at that point as well, with $\mu=0$.

Given this setup, the Grossman-Hart conditions for $s^{*}(\cdot)$ to be nondecreasing concave are simply:

(iia) $f_{a}(\mathbf{x} \mid a) / f(\mathbf{x} \mid a)$ nondecreasing concave in $\mathbf{x}$ for each $a$, and

(iib) $1 / u^{\prime}(s)$ increasing convex in $s$ so $\nu(z)=\left(u^{\prime}\right)^{-1}(1 / z)$ increasing concave in $z$. Condition (iia) strengthens the Monotone Likelihood Ratio property. It says that the information in $\tilde{\mathbf{x}}$ grows more and more slowly as $\tilde{\mathbf{x}}$ rises, which tends to make $s^{*}(\mathbf{x})$ concave. Condition (iib) says that the marginal utility of income falls quickly so, for $s$ large, further increases in $s$ have less effect in terms of incentives. This also tends to make $s^{*}(\mathbf{x})$ concave. Thus, (iia) and (iib) are clearly relevant for $s^{*}(\cdot)$ to be concave.

The Grossman-Hart conditions, plus $\mu \geq 0$, ensure that the function

$$
s^{*}(\mathbf{x})=\nu\left(\lambda+\mu\left[f_{a}\left(\mathbf{x} \mid a^{*}\right) / f\left(\mathbf{x} \mid a^{*}\right)\right]\right)
$$

is nondecreasing concave in $\mathbf{x}$. This combined with Conditions (i) and (iii), ensure that the first-order approach is valid. Thus, let $L_{a^{*}}(\mathbf{x})=\lambda+\mu\left[f_{a}\left(\mathbf{x} \mid a^{*}\right) / f\left(\mathbf{x} \mid a^{*}\right)\right]$ be 
the likelihood ratio, evaluated at $a^{*}$, and adjusted by the multipliers $\lambda$ and $\mu$. Then $U\left(s^{*}(\cdot), a\right)$ is the expectation of the following composition of functions:

$$
u\left(\nu\left(L_{a^{*}}(\mathbf{x}(\vartheta, a))\right)\right)=u \circ \nu \circ L_{a^{*}} \circ \mathbf{x}(a, \vartheta)
$$

Conditions (i), (iia), (iib) and (iii) then clearly imply that (9) is concave in $a$ for each $\vartheta$. Thus, $U\left(s^{*}(\cdot), a\right)=E\left[u\left(\nu\left(L_{a^{*}}(\mathbf{x}(a, \tilde{\vartheta}))\right)\right)\right]-a$ is concave in $a$ as well.

Moreover, thinking of $u \circ \nu \circ L_{a^{*}} \circ \mathbf{x}$ as $(u \circ \nu) \circ L_{a^{*}} \circ \mathbf{x}$ shows that Conditions (iib) and (iii) can be combined and generalized, since what matters is for the composition of $\nu$ and $u$ to be concave, not for each to be concave separately. But this composition is simply Jewitt's function

$$
\omega(z)=u(\nu(z))=u\left(\left(u^{\prime}\right)^{-1}(1 / z)\right)
$$

so we can replace (iib) and (iii) with Jewitt's condition that $\omega(z)$ is concave in $z .^{6}$

Proposition 2: Assume (a) the coordinates of $\mathbf{x}(a, \vartheta)$ are concave in $a$, (b) $f_{a}(\mathbf{x} \mid a) / f(\mathbf{x} \mid a)$ is nondecreasing concave in $\mathbf{x}$, and (c) $\omega(z)$ is increasing concave in $z$. Then any solution to the relaxed problem, maximizing $V(s(\cdot)$, a) subject to (5) and (6), also solves the full problem of maximizing $V(s(\cdot)$, a) subject to (4) and (5).

PROOF: Conditions (a), (b) and (c) imply that any solution to the relaxed problem yields an agent objective function $U\left(s^{*}(\cdot), a\right)$, from $\left(3_{\mathrm{SS}}\right)$, which is concave in $a$.

6 This condition makes considerable sense if viewed through the dual representation of the principal-agent problem. That is, if we let $\psi(z)=\max _{s} z u(s)-s$ be a profit function from "selling utility" at shadow price $z$, then concavity of $\omega(z)$ can be expressed as $\psi^{\prime \prime \prime}(z) \leq 0$ (Jewitt (2007), footnote 18; see also Fagart and Sinclair-Desgagné (2007), who explore further economic implications of $\psi^{\prime \prime \prime}(z)$ positive or negative).

Intuitively, $s^{*}=\nu(z)$ solves $z u^{\prime}\left(s^{*}\right)=1$, so it maximizes $z u(s)-s$. Thus, by Hotelling's lemma, $\psi^{\prime}(z)=u\left(s^{*}\right)=u(\nu(z))=\omega(z)$ is the "supply" of utility as a function of its shadow price $z$, so $\omega^{\prime \prime}(z)=\psi^{\prime \prime \prime}(z) \leq 0$ implies that this supply curve is concave in the shadow price. Then, if the shadow price $z=L_{a^{*}}(\mathbf{x}(a, \vartheta))$ is concave in $a$ for each $\vartheta, U\left(s^{*}(\cdot), a\right)$ is concave in $a$ also. 
Since $U\left(s^{*}(\cdot), a\right)$ is concave, and $U_{a}\left(s^{*}(\cdot), a^{*}\right)=0, a^{*}$ also satisfies the full incentive compatibility constraint (4), so it is a solution to the fully constrained problem. Q.E.D.

Proposition 2 is similar to Jewitt's (1988) Theorem 3, but without his independence assumption. The proof is also quite similar. Jewitt (1988), footnote 6, mentions that "[i]t is possible to derive some conditions without [independence], but we do not pursue the issue here." Thus, Jewitt could clearly have obtained something like our Proposition 2. However, it is not immediately obvious how to express the condition, that $\mathbf{x}(a, \vartheta)$ is concave in $a$, using the Mirrlees notation.

To translate this condition into the Mirrlees notation, we must begin with a Mirrlees representation, $f(\mathbf{x} \mid a)$, and find a state-space model that yields this density. We then impose concavity of $\mathbf{x}(a, \vartheta)$ in the state-space model, and translate back to the Mirrlees notation. Thus, for the random vector $\tilde{\mathbf{x}}$ with density $f(\mathbf{x} \mid a)$, we need a representation $x_{i}(a, \tilde{\vartheta}), i=1,2, \ldots, n$, where $\tilde{\vartheta}$ has some joint density $p(\vartheta)$ independent of $a$, such that the random variables $x_{i}(a, \tilde{\vartheta})$ have joint density $f(\mathbf{x} \mid a)$.

There are many such representations, and each provides different sufficient conditions. This is somewhat inelegant, since there is no one canonical representation. It may, however, supply some flexibility. If one representation does not work, the researcher can try another. The representation in Proposition 3 builds on what is known as the "standard construction" (see Müller and Stoyan (2002, p. 88)).

Consider the two signal case for specificity (the approach clearly generalizes), let $F^{1}\left(x_{1} \mid a\right)$ be the marginal cumulative distribution function of $\tilde{x}_{1}$ as a function of $a$, and let $F^{2}\left(x_{2} \mid x_{1}, a\right)$ be the conditional cumulative distribution function of $\tilde{x}_{2}$, given $\tilde{x}_{1}=x_{1}$, as a function of $a$. We construct one representation, and then indicate how others might be obtained.

Proposition 3: Suppose that $F^{1}$ and $F^{2}$ are continuous, and that the support 
of the distribution of $\tilde{\mathbf{x}}$ is compact and convex. Then there exist continuous functions $x_{1}\left(a, \theta_{1}, \theta_{2}\right)$ and $x_{2}\left(a, \theta_{1}, \theta_{2}\right)$, for $\left(\theta_{1}, \theta_{2}\right) \in[0,1] \times[0,1]$, that solve the system of equations

$$
F^{1}\left(x_{1} \mid a\right)=\theta_{1}, \quad F^{2}\left(x_{2} \mid x_{1}, a\right)=\theta_{2} .
$$

Also, if $\tilde{\vartheta}=\left(\tilde{\theta}_{1}, \tilde{\theta}_{2}\right)$ is uniformly distributed on the square $[0,1] \times[0,1]$, then $\mathbf{x}(a, \tilde{\vartheta})$ $=\left(x_{1}(a, \tilde{\vartheta}), x_{2}(a, \tilde{\vartheta})\right)$ has the same joint distribution as $\tilde{\mathbf{x}}$.

ProOF: First, $F^{1}\left(x_{1} \mid a\right)$ is continuous, and also increasing in $x_{1}$ on its (convex) support. Thus the function $x_{1}\left(a, \theta_{1}, \theta_{2}\right)=\left(F^{1}\right)^{-1}\left(\theta_{1} \mid a\right)$, solving the first half of (11), exists and is continuous (and depends only on $\theta_{1}$ and $a$ ). Next, plug $x_{1}=\left(F^{1}\right)^{-1}\left(\theta_{1} \mid a\right)$ into the second half of (11). Then one can similarly solve $F^{2}\left(x_{2} \mid\left(F^{1}\right)^{-1}\left(\theta_{1} \mid a\right), a\right)=\theta_{2}$ for $\left(F^{2}\right)^{-1}\left(\theta_{2} \mid\left(F^{1}\right)^{-1}\left(\theta_{1} \mid a\right), a\right)=x_{2}\left(a, \theta_{1}, \theta_{2}\right)$.

Now, $\operatorname{Prob}\left(x_{1}\left(a, \tilde{\theta}_{1}, \tilde{\theta}_{2}\right) \leq x_{1}\right)=\operatorname{Prob}\left(\left(F^{1}\right)^{-1}\left(\tilde{\theta}_{1} \mid a\right) \leq x_{1}\right)=\operatorname{Prob}\left(\tilde{\theta}_{1} \leq F^{1}\left(x_{1} \mid a\right)\right)$ $=F^{1}\left(x_{1} \mid a\right)$, since $\tilde{\theta}_{1}$ is $U[0,1]$. A similar logic shows that $\operatorname{Prob}\left(x_{2}\left(a, \tilde{\theta}_{1}, \tilde{\theta}_{2}\right) \leq x_{2} \mid\right.$ $\left.x_{1}\left(a, \tilde{\theta}_{1}, \tilde{\theta}_{2}\right)=x_{1}\right)=F^{2}\left(x_{2} \mid x_{1}, a\right) . \quad$ Q.E.D.

Proposition 3 lets us check concavity of $\mathbf{x}(a, \vartheta)$ in the context of a Mirrlees framework. Thus, suppose that $\left(F^{1}\right)^{-1}\left(\theta_{1} \mid a\right)$ is concave in $a$ and that $\left(F^{2}\right)^{-1}\left(\theta_{2} \mid x_{1}, a\right)$ is nondecreasing in $x_{1}$ and concave in $x_{1}$ and $a$. Then $\mathbf{x}(a, \vartheta)$ from Proposition 3 is concave in $a$. This, plus $f_{a}(\mathbf{x} \mid a) / f(\mathbf{x} \mid a)$ nondecreasing concave in $\mathbf{x}$ and $\omega(z)$ increasing concave in $z$, is sufficient to support the first order approach, by Proposition 2.

Other representations are also possible. For example, the roles of $x_{1}$ and $x_{2}$ could be switched above. More generally, if one considers a density-preserving smooth deformation, $\Phi_{a}(\vartheta)=\vartheta^{\prime}$, of the square $[0,1] \times[0,1]$, then $\tilde{\mathbf{x}}=\mathbf{x}\left(a, \Phi_{a}(\tilde{\vartheta})\right)$ gives a new state-space representation of the same Mirrlees model $f(\mathbf{x} \mid a)$.

Jewitt (1988), in his Theorem 3, assumes $\tilde{x}_{1}$ and $\tilde{x}_{2}$ are independent from one another, so the functions in Proposition 3 reduce to $x_{i}\left(a, \theta_{i}\right)=\left(F^{i}\right)^{-1}\left(\theta_{i} \mid a\right)$. He 
also assumes that the $F^{i}\left(x_{i} \mid a\right)$ are quasiconvex in $x_{i}$ and $a$. This is how he imposes concavity of the $x_{i}\left(a, \theta_{i}\right)$ in $a$. His Theorem 3 then assumes this plus $f_{a}(\mathbf{x} \mid a) / f(\mathbf{x} \mid a)$ nondecreasing concave in $\mathbf{x}$, and $\omega(z)$ concave in $z$, as in our Proposition 2.

To check concavity of $\mathbf{x}(a, \vartheta)$ more generally, one can implicitly differentiate (11) twice to obtain $\partial^{2} x_{i} / \partial a^{2}$, and check concavity through the analogue of bordered Hessians. However, this may be computationally messy. It might therefore be easier to use a shortcut to check concavity of $\mathbf{x}(a, \vartheta)$. For example, one might begin with a state-space representation, or solve (11) explicitly (see Conlon (2008)).

\section{A MULTi-SIGNAL EXTENSION OF THE CDFC}

As suggested in Section 3 above, if we express the agent's payoff function using the Mirrlees formalism, $\left(3_{\mathrm{M}}\right)$, (i.e., $\left.U\left(s^{*}(\cdot), a\right)=\int u\left(s^{*}(\mathbf{x})\right) f(\mathbf{x} \mid a) d \mathbf{x}-a\right)$, then we are naturally led to impose concavity through the density function, $f(\mathbf{x} \mid a)$, since that is where the agent's choice variable $a$ appears. Consider, for example, the one-signal case, so $\tilde{\mathbf{x}}$ reduces to the univariate signal $\tilde{x}$, and let the support of $f(x \mid a)$ be $[\underline{x}, \bar{x}]$. Then integrating by parts gives

$$
U\left(s^{*}(\cdot), a\right)=u\left(s^{*}(\underline{x})\right)+\int_{\underline{x}}^{\bar{x}} \frac{d u\left(s^{*}(x)\right)}{d x}[1-F(x \mid a)] d x-a .
$$

Thus, suppose $u\left(s^{*}(x)\right)$ is nondecreasing in $x$ (applying, e.g., the monotone likelihood ratio property), so $d u\left(s^{*}(x)\right) / d x \geq 0$. Rogerson's Convexity of the Distribution Function Condition (CDFC) states that $F(x \mid a)$ is convex in $a$. This implies that $1-F(x \mid a)$ is concave in $a$, so (12) clearly implies that $U\left(s^{*}(\cdot), a\right)$ is concave in $a .^{7}$

7 Note that a new derivation of Jewitt's (1988) full one-signal conditions can be obtained similarly, by performing a second integration by parts on (12). Thus, if we let $\hat{F}(x \mid a)=\int_{\underline{x}}^{x}[1-F(z \mid a)] d z$, then a second integration by parts yields $U\left(s^{*}(\cdot), a\right)$ $=u\left(s^{*}(\underline{x})\right)+\left.\left(d u\left(s^{*}(x)\right) / d x\right)\right|_{\bar{x}} \hat{F}(\bar{x} \mid a)-\int_{\underline{x}}^{\bar{x}}\left(d^{2} u\left(s^{*}(x)\right) / d x^{2}\right) \hat{F}(x \mid a) d x-a$. Jewitt 
The natural multi-signal generalization of (12) would be to choose one of the $n$ signals, say $x_{h}$, and integrate $\left(3_{\mathrm{M}}\right)$ by parts with respect to $x_{h}$. This yields essentially the GCDFC of Sinclair-Desgagné (1994).

To obtain a more flexible generalization of the CDFC, begin by returning to the one-signal case, and note that, if $u\left(s^{*}(x)\right)$ is nondecreasing in $x$, then it can clearly be approximated by a sum of nondecreasing step functions. Specifically, it can be approximated as a sum, $u\left(s^{*}(x)\right) \approx \alpha_{0}+\sum_{j \geq 1} \alpha_{j} h\left(x ; b_{j}\right)$, where the $\alpha_{j}$ 's are positive for $j \geq 1$, and $h(x ; b)$ is the step function which is zero for $x<b$ and one for $x \geq b$. The integral $\int u\left(s^{*}(x)\right) f(x \mid a) d x$ in $U\left(s^{*}(\cdot), a\right)$ can then be approximated as

$$
\int u\left(s^{*}(x)\right) f(x \mid a) d x \approx \alpha_{0}+\sum_{j \geq 1} \alpha_{j} \int h\left(x ; b_{j}\right) f(x \mid a) d x=\alpha_{0}+\sum_{j \geq 1} \alpha_{j}\left[1-F\left(b_{j} \mid a\right)\right] .
$$

By the CDFC this sum is concave in $a$, so $U\left(s^{*}(\cdot), a\right)$ is concave in $a$, as desired.

To obtain a new multi-signal generalization of the CDFC approach, we must therefore simply construct a family of multi-variable generalizations of the functions $h(x ; b)$ above, capable of generating all multi-variable nondecreasing functions.

Thus, consider the $n$ signal case, and say that the set $\mathbf{E} \subseteq \mathbf{R}^{n}$ is an increasing set (Nachbin (1965), Milgrom and Weber (1982)) if $\mathbf{x} \in \mathbf{E}$ and $\mathbf{y} \geq \mathbf{x}$ (i.e., $y_{i} \geq x_{i}, i=$ $1,2, \ldots, n)$, implies $\mathbf{y} \in \mathbf{E}$. Note that if a function is nondecreasing, then its upper level sets are increasing sets. Given an increasing set $\mathbf{E}$, let $h(\mathbf{x} ; \mathbf{E})$ be the characteristic

assumes that $f_{a}(x \mid a) / f(x \mid a)$ is nondecreasing concave in $x$, and $\omega(z)$ is increasing concave in $z$, which imply $d u\left(s^{*}(x)\right) / d x \geq 0$ and $d^{2} u\left(s^{*}(x)\right) / d x^{2} \leq 0$, as in Proposition 2 above. An additional condition of his, that $\int_{\underline{x}}^{x} F(z \mid a) d z$ is convex in $a$, yields $\hat{F}(x \mid a)$ concave in $a$. This implies that $U\left(s^{*}(\cdot), a\right)$ above is concave in $a$, so the first order approach applies. In the current one signal case, Jewitt's condition, $\int_{\underline{x}}^{x} F(z \mid a) d z$ convex in $a$, generalizes Proposition 2's concavity in $a$ of $\mathbf{x}(a, \vartheta)$. However, there is no simple multi-signal version of $\int_{\underline{x}}^{x} F(z \mid a) d z$ convex in $a$ (see Conlon (2008)). 
function of $\mathbf{E}$, so

$$
h(\mathbf{x} ; \mathbf{E})=\left\{\begin{array}{l}
0 \text { if } \mathbf{x} \notin \mathbf{E} \\
1 \text { if } \mathbf{x} \in \mathbf{E}
\end{array}\right.
$$

Then every nondecreasing function can be approximated using positive linear combinations of these $h(\mathbf{x} ; \mathbf{E})$ functions, yielding a multi-signal extension of the above approach. This suggests the following generalization of the CDFC:

Definition 1: The distribution $f(\mathbf{x} \mid a)$ satisfies the Concave Increasing-Set Probability (CISP) condition if, for every increasing set $\mathbf{E}$, the probability $\operatorname{Prob}(\tilde{\mathbf{x}} \in \mathbf{E} \mid a)$ is concave in $a$.

Now the CISP condition, plus the Monotone Likelihood Ratio (MLR) property $\left(f_{a}(\mathbf{x} \mid a) / f(\mathbf{x} \mid a)\right.$ nondecreasing in $\left.\mathbf{x}\right)$ and strict concavity of $u(\cdot)$, can be used to justify the first-order approach. To see this, begin with a lemma.

LEMMA 1: The density $f(\cdot \mid a)$ satisfies CISP if and only if the transformation

$$
\varphi^{T}(a)=\int \varphi(\mathbf{x}) f(\mathbf{x} \mid a) d \mathbf{x}=E[\varphi(\tilde{\mathbf{x}}) \mid a]
$$

satisfies:

$$
\text { for any nondecreasing function } \varphi(\mathbf{x}), \varphi^{T}(a) \text { is concave in a. }
$$

PROOF: The proof builds on a standard approximation (see, e.g., Müller and Stoyan (2002), Theorem 3.3.4), and is contained in the appendix.

With this lemma in hand, it is easy to give conditions justifying the first-order approach in the multi-signal case. This is done in the following proposition.

Proposition 4: Suppose that $u(\cdot)$ is increasing and strictly concave, and that the MLR and CISP conditions hold. Then any solution to the relaxed principal-agent problem also solves the fully constrained problem. 
Proof: First, the solution to the relaxed problem has $s^{*}(\mathbf{x})$ solving (7). Also, since $u(\cdot)$ is strictly concave, $1 / u^{\prime}(s)$ is increasing in $s$. This, plus $\mu \geq 0$ (by the Jewitt (1988) argument), and $f_{a}(\mathbf{x} \mid a) / f(\mathbf{x} \mid a)$ nondecreasing in $\mathbf{x}$, implies that the payment schedule $s^{*}(\mathbf{x})$ is also nondecreasing in $\mathbf{x}$. Thus $u\left(s^{*}(\mathbf{x})\right)$ is nondecreasing in $\mathbf{x}$. Also, letting $\varphi(\mathbf{x}) \equiv u\left(s^{*}(\mathbf{x})\right)$ gives $U\left(s^{*}(\cdot), a\right)=\varphi^{T}(a)-a$, by $\left(3_{\mathrm{M}}\right)$ and (13). Using this and CISP in Lemma 1 then implies that $U\left(s^{*}(\cdot), a\right)$ is concave in $a$, so any solution to the relaxed problem is also a solution to the fully constrained problem. Q.E.D.

\section{A RISK AVERSE PRINCIPAL}

Up to now, the principal has been assumed risk neutral. This was primarily because the Jewitt conditions go through more smoothly in this case. However, suppose now that the principal is risk averse, with von Neumann-Morgenstern utility function $v(\cdot)$. Then the principal's first order condition for $s^{*}(\cdot),(7)$, changes to

$$
\frac{v^{\prime}\left(\pi(\mathbf{x})-s^{*}(\mathbf{x})\right)}{u^{\prime}\left(s^{*}(\mathbf{x})\right)}=\lambda+\mu \frac{f_{a}\left(\mathbf{x} \mid a^{*}\right)}{f\left(\mathbf{x} \mid a^{*}\right)} .
$$

Thus, even if we assume that the likelihood ratio $f_{a}\left(\mathbf{x} \mid a^{*}\right) / f\left(\mathbf{x} \mid a^{*}\right)$ is concave in $\mathbf{x}$, it is difficult to impose the nonstandard condition $s^{*}(\mathbf{x})$ concave in $\mathbf{x}$, because it is no longer enough to assume that $\nu(z)=\left(u^{\prime}\right)^{-1}(1 / z)$ is concave. Therefore Jewitt's condition, concavity of $\omega(z)$, is also inadequate.

On the other hand, the CDFC approach, and its generalization in Section 5, are easily adapted to a risk averse principal. This requires two new assumptions, i.e., that the function $\pi(\mathbf{x})$ is nondecreasing in $\mathbf{x}$, and that $f(\mathbf{x} \mid a)$ satisfies the NISP condition, defined as follows:

DEFINITION 2: The distribution $f(\mathbf{x} \mid a)$ satisfies the Nondecreasing Increasing-Set Probability (NISP) condition if, for every increasing set $\mathbf{E}$, the probability $\operatorname{Prob}(\tilde{\mathbf{x}} \in$ $\mathbf{E} \mid a)$ is nondecreasing in $a$. 
Now, Jewitt's (1988) proof of $\mu \geq 0$ does not work if the principal is risk averse. One must therefore replace (6) with Rogerson's (1985) doubly relaxed constraint,

$$
U_{a}\left(s^{*}(\cdot), a^{*}\right) \geq 0 .
$$

The principal's "doubly relaxed" problem is then to maximize $V(s(\cdot), a)$ given the participation constraint (5) and the doubly relaxed incentive compatibility constraint (16). This leads to the following proposition:

Proposition 5: Assume $\pi(\cdot)$ is nondecreasing, $u(\cdot)$ and $v(\cdot)$ are increasing and strictly concave, and the MLR, CISP, and NISP conditions hold. Then any solution to the principal's doubly relaxed problem is also a solution to her unrelaxed problem.

ProOF: Let $s^{*}(\cdot)$ and $a^{*}$ be solutions to the doubly relaxed problem. As usual, (15) still holds. Also, since (16) is an inequality constraint, $\mu \geq 0$. This, plus $u(\cdot)$ and $v(\cdot)$ strictly concave, MLR, and $\pi(\mathbf{x})$ nondecreasing, imply $s^{*}(\mathbf{x})$ nondecreasing.

If $U_{a}\left(s^{*}(\cdot), a^{*}\right)>0$, then $a^{*}$ is not incentive compatible. Thus, we must show that this contradicts the assumption that $s^{*}(\cdot)$ and $a^{*}$ solve the doubly relaxed problem.

Suppose therefore that $U_{a}\left(s^{*}(\cdot), a^{*}\right)>0$. Then $\mu=0$, so (15) shows that $v^{\prime}(\pi(\mathbf{x})-$ $\left.s^{*}(\mathbf{x})\right) / u^{\prime}\left(s^{*}(\mathbf{x})\right)$ is constant. Thus $s^{*}(\mathbf{x})$ nondecreasing in $\mathbf{x}$ implies that $\pi(\mathbf{x})-s^{*}(\mathbf{x})$ is nondecreasing in $\mathbf{x}$, so $\varphi(\mathbf{x}) \equiv v\left(\pi(\mathbf{x})-s^{*}(\mathbf{x})\right)$ is also nondecreasing in $\mathbf{x}$. Therefore, by an argument like that in the proof of Lemma 1 above, NISP implies that the principal's payoff, $V\left(s^{*}(\cdot), a\right)=\varphi^{T}(a)=\int v\left(\pi(\mathbf{x})-s^{*}(\mathbf{x})\right) f(\mathbf{x} \mid a) d \mathbf{x}$, is nondecreasing in $a$. This, plus $U_{a}\left(s^{*}(\cdot), a^{*}\right)>0$, implies that $s^{*}(\cdot)$ and $a^{*}$ are not solutions to the doubly relaxed problem, since increasing $a$ and shifting the $s^{*}(\cdot)$ schedule down slightly can increase the principal's payoff, while still satisfying the constraints. Thus $U_{a}\left(s^{*}(\cdot), a^{*}\right)>0$ leads to a contradiction, so $U_{a}\left(s^{*}(\cdot), a^{*}\right)=0$.

Next, since $s^{*}(\cdot)$ is nondecreasing, $u\left(s^{*}(\cdot)\right)$ is nondecreasing, so CISP, plus Lemma 
1 above, imply that the agent's objective function is concave. Thus $s^{*}(\cdot)$ and $a^{*}$ solve the unrelaxed problem and the first-order approach is valid.

Q.E.D.

As Ian Jewitt has pointed out to me, NISP can be checked by adapting a version of Milgrom and Weber's (1982) notion of affiliated random variables (equivalently $\mathrm{MPT}_{2}$; see Müller and Stoyan (2002, pp. 126-7)). Thus, assume $f(\mathbf{x} \mid a)$ is smooth in $(\mathbf{x}, a)$, and that $a \in[0,1]$. Though $a$ is not random, imagine it is a random variable, with marginal distribution uniform on $[0,1]$. Then the random vector $(\tilde{\mathbf{x}}, \tilde{a})$, with joint density $f^{\#}(\mathbf{x}, a)=f(\mathbf{x} \mid a)$, is affiliated if the cross partials $\partial^{2} \ln f^{\#} / \partial x_{i} \partial a$ and $\partial^{2} \ln f^{\#} / \partial x_{i} \partial x_{j}$ are all nonnegative. NISP then follows immediately from Milgrom and Weber's Theorem 5. Note, incidentally, that $\partial^{2} \ln f^{\#} / \partial x_{i} \partial a \geq 0$ is the MLR property.

Unfortunately, it is not clear how this approach could be used to check CISP. The next two sections therefore use a different approach to derive conditions ensuring CISP. While affiliation is sufficient to ensure NISP, the approach in the following sections also gives alternative conditions ensuring NISP, with little extra effort. Also, these alternative conditions are less restrictive than affiliation.

\section{REDUCING NISP AND CISP TO DENSITY FLOWS}

The previous sections developed the CISP condition, which generalizes the CDFC. CISP is also less restrictive than the GCDFC. As a referee has pointed out, GCDFC implies that there is an index $h$ such that $\operatorname{Prob}(\tilde{\mathbf{x}} \in \mathbf{E} \mid a)$ is concave in $a$ for all sets $\mathbf{E}$ satisfying the condition: $\left(x_{h}, \mathbf{x}_{-h}\right) \in \mathbf{E}$ and $x_{h}^{\prime} \geq x_{h}$ imply $\left(x_{h}^{\prime}, \mathbf{x}_{-h}\right) \in \mathbf{E}$. Since this class of sets is larger than the class of increasing sets, GCDFC is more restrictive than CISP. However, it remains to see more clearly just how flexible CISP is.

To construct distributions satisfying CISP, one can think of $f(\mathbf{x} \mid a)$ in terms of probability density flows as $a$ increases. Analyzing these flows, in turn, requires tools 
from vector calculus (Taylor and Mann (1983), Chapter 15; Lang (1987), Chapters 10 and 12). For expositional purposes focus on the two-signal case, though the approach generalizes easily to $n$ signals.

Thus, consider the random vector $\tilde{\mathbf{x}}=(\tilde{x}, \tilde{y})$ on the square $\mathbf{S}=[0,1] \times[0,1]$, with density $f(x, y \mid a)=f(\mathbf{x} \mid a)$. Also, consider a vector field $\mathbf{v}(\mathbf{x}, a)=(u(\mathbf{x}, a), v(\mathbf{x}, a))$ on $\mathbf{S}$. If the mass of $f(\mathbf{x} \mid a)$ moves along the vector field $\mathbf{v}(\mathbf{x}, a)$ as $a$ grows, then

$$
f_{a}(x, y \mid a)=-\left[u_{x}(x, y, a)+v_{y}(x, y, a)\right]=-\operatorname{div} \mathbf{v}(\mathbf{x}, a) .
$$

This is the usual "divergence" formula for the flow of a compressible fluid, where $\mathbf{v}(\mathbf{x}, a)$ is the "flux" (density $\times$ velocity of flow). Thus if, e.g., $u_{x}\left(x_{0}, y_{0}, a\right)>0$, then $u\left(x_{0}-\Delta x, y_{0}, a\right)<u\left(x_{0}+\Delta x, y_{0}, a\right)$ so, in the $x$-direction, less mass is moving towards $\left(x_{0}, y_{0}\right)$ than away from it. That is, the mass does diverge. This should make $f\left(x_{0}, y_{0} \mid a\right)$ fall in $a$, as in (17). A similar argument applies for the $y$-direction. This therefore does represent $f(x, y \mid a)$ in terms of a density flow, as expected.

Next let $\mathbf{A}$ be a simply connected two-dimensional subset of $\mathbf{S}$ with boundary $\partial \mathbf{A}$, and let $\partial \mathbf{A}$ be traced out counterclockwise by the continuous piecewise differentiable simple closed curve $\mathbf{x}(t)$ as $t$ rises from zero to one. Let $\mathbf{n}(t)=\left(y^{\prime}(t),-x^{\prime}(t)\right)$ be an outward pointing "normal" (perpendicular) vector to $\partial \mathbf{A}$ at $\mathbf{x}(t)$, rotated clockwise $90^{\circ}$ from the tangent vector $\mathbf{x}^{\prime}(t)=\left(x^{\prime}(t), y^{\prime}(t)\right)$ (see Figure 1). Then

$$
\begin{aligned}
& \frac{d}{d a} \iint_{\mathbf{A}} f(\mathbf{x} \mid a) d \mathbf{x}=-\iint_{\mathbf{A}}\left[u_{x}(x, y, a)+v_{y}(x, y, a)\right] d x d y \\
& \quad=-\int_{0}^{1}\left[u(\mathbf{x}(t), a) y^{\prime}(t)-v(\mathbf{x}(t), a) x^{\prime}(t)\right] d t=-\int_{0}^{1} \mathbf{v}(\mathbf{x}(t), a) \cdot \mathbf{n}(t) d t .
\end{aligned}
$$

Here $\iint_{\mathbf{A}}$ denotes a double integral over the area $\mathbf{A}$. In addition, the first step of (18) follows from (17), and the second step follows from the two dimensional "Divergence 
Theorem" (Taylor and Mann (1983, p. 488), Lang (1987, pp. 285, 345)). Finally, $\mathbf{v}(\mathbf{x}(t), a) \cdot \mathbf{n}(t)=u(\mathbf{x}(t), a) y^{\prime}(t)-v(\mathbf{x}(t), a) x^{\prime}(t)$ is the "dot product" of the vectors $\mathbf{v}(\mathbf{x}(t), a)$ and $\mathbf{n}(t)$.

This dot product is positive if $\mathbf{v}(\mathbf{x}(t), a)$ points out from the boundary of $\mathbf{A}$, and negative if it points into A. For example, at the point $\mathbf{x}(t)$ in Figure $1, \mathbf{v}(\mathbf{x}(t), a)$ is pointing into $\mathbf{A}$, so $\mathbf{v}(\mathbf{x}(t), a) \cdot \mathbf{n}(t)<0$, which makes a positive contribution to (18), increasing the mass inside $\mathbf{A}$, as it should. Thus the last integral in (18) is the flow of the vector field $\mathbf{v}(\mathbf{x}(t), a)$ across the boundary $\partial \mathbf{A}$, so (18) gives the density flow of $\mathbf{v}(\mathbf{x}, a)$ into $\mathbf{A}$. The equation thus makes intuitive sense.

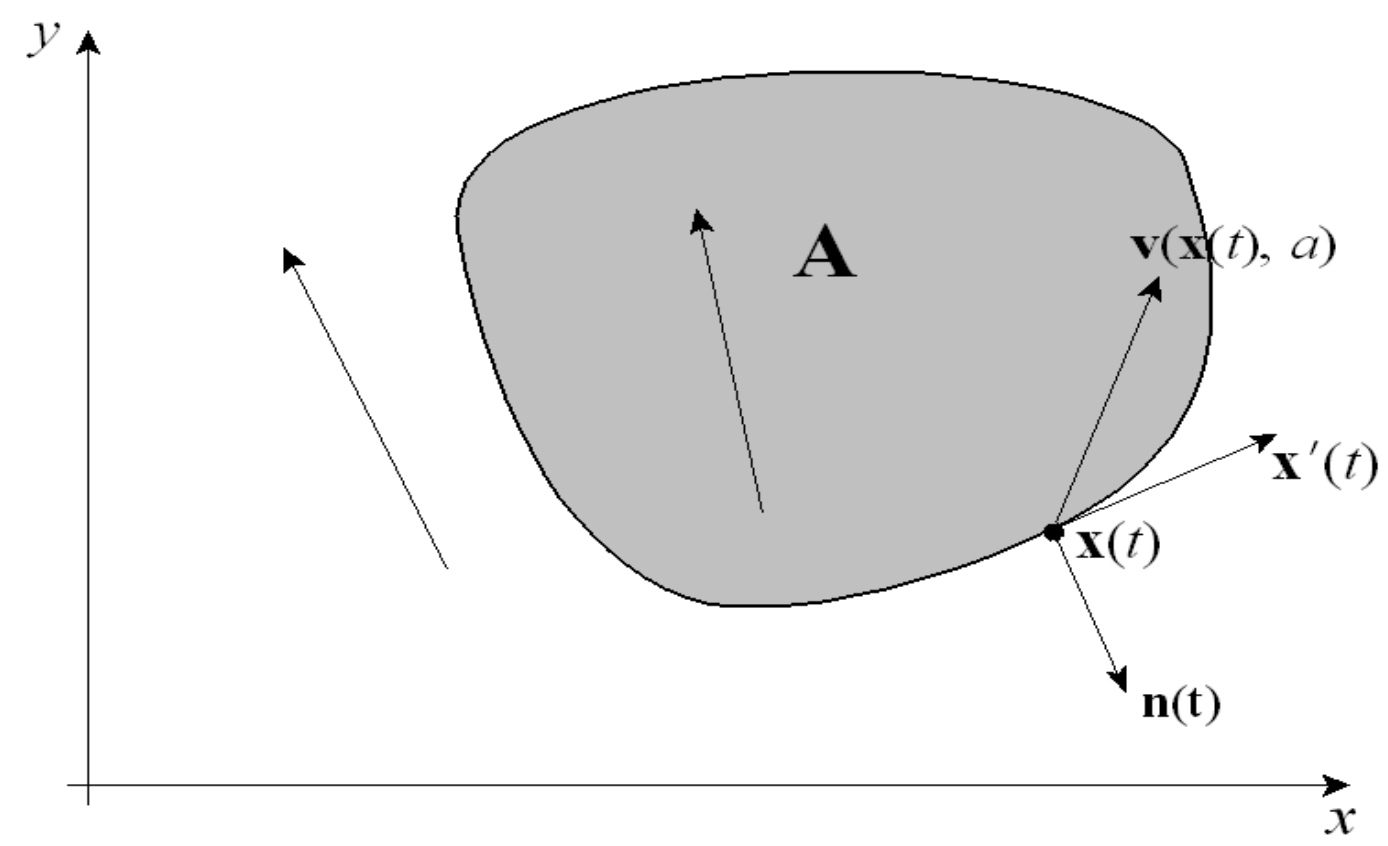

FIGURE 1.-The vector flow into region $\mathbf{A}$. The normal vector $\mathbf{n}(t)$, at the point $\mathbf{x}(t)$, is the vector pointing out from $\mathbf{A}$, perpendicular to the tangent vector $\mathbf{x}^{\prime}(t)$. At the point shown, there is an obtuse angle between $\mathbf{n}(t)$ and the density flux $\mathbf{v}(\mathbf{x}(t), a)$. The dot product $\mathbf{v}(\mathbf{x}(t), a) \cdot \mathbf{n}(t)$ is therefore negative, so the contribution to the aggregate flow into A, given in Equation (18), is positive, as it should be.

To illustrate (18) we show that $f(\mathbf{x} \mid a)$ from (17) is a probability density function on the square $\mathbf{S}$ for all $a$ if it is a probability density function on $\mathbf{S}$ for $a=0$, and if in 
addition $f(\mathbf{x} \mid a)$ stays nonnegative and the boundary conditions

$$
u(0, y, a)=u(1, y, a)=v(x, 0, a)=v(x, 1, a)=0
$$

hold. The conditions in (19) ensure that no mass is flowing across the boundaries of the square $\mathbf{S}$. To see that this works, translate into ordinary multiple integrals:

$$
\begin{aligned}
\frac{d}{d a} & \int_{0}^{1} \int_{0}^{1} f(x, y \mid a) d x d y=-\int_{0}^{1} \int_{0}^{1}\left[u_{x}(x, y, a)+v_{y}(x, y, a)\right] d x d y \\
\quad & =-\int_{0}^{1}[u(1, y, a)-u(0, y, a)] d y-\int_{0}^{1}[v(x, 1, a)-v(x, 0, a)] d x=0 .
\end{aligned}
$$

Here the first step uses (17), the second step uses the Fundamental Theorem of Calculus and the last step uses (19). Thus $\iint_{\mathbf{S}} f(\mathbf{x} \mid a) d \mathbf{x}$ is constant in $a$, so if $\iint_{\mathbf{S}} f(\mathbf{x} \mid 0) d \mathbf{x}=1$, then $\iint_{\mathbf{S}} f(\mathbf{x} \mid a) d \mathbf{x}=1$ for all $a$ and, assuming $f(\mathbf{x} \mid a) \geq 0, f(\mathbf{x} \mid a)$ really is a probability density function on $\mathbf{S}$. The third expression in (20), incidentally, is the analogue for the square, $\mathbf{S}$, of the last two expressions in (18). Thus (20) gives a proof of a special case of (18). Note that this calculation suggests that the Divergence Theorem is a generalization of the Fundamental Theorem of Calculus.

Integrating (17), finally, gives:

$$
f(x, y \mid a)=f(x, y \mid 0)-\int_{0}^{a}\left[u_{x}(x, y, \alpha)+v_{y}(x, y, \alpha)\right] d \alpha .
$$

With this machinery, it is easy to construct densities which satisfy NISP and CISP. We show how to do this in the following lemma.

LEMMA 2 (THE DENSITY FLOW LEMMA): Let the vector field $\mathbf{v}(\mathbf{x}$, a) satisfy (19). Then if its coordinates are nonnegative, the density function $f(x, y \mid a)$ in (21) satisfies NISP. Similarly, if its coordinates are nonincreasing in $a$, then $f(x, y \mid a)$ satisfies CISP.

PROOF: Let E be an increasing set. Using (18) gives

$$
\frac{d}{d a} \operatorname{Prob}(\tilde{\mathbf{x}} \in \mathbf{E} \mid a)=\frac{d}{d a} \operatorname{Prob}(\tilde{\mathbf{x}} \in \mathbf{E} \cap \mathbf{S} \mid a)=-\int_{0}^{1} \mathbf{v}(\mathbf{x}(t), a) \cdot \mathbf{n}(t) d t
$$


with $\mathbf{x}(t)$ tracing $\partial(\mathbf{E} \cap \mathbf{S})$. Since $\mathbf{E}$ is an increasing set, the boundary $\partial(\mathbf{E} \cap \mathbf{S})$ consists of a downward sloping curve on its Southwest, whose normal vector $\mathbf{n}(t)$ has nonpositive coordinates, and other lines, from the boundary of $\mathbf{S}$. Now, if the vector field $\mathbf{v}(\mathbf{x}, a)$ has nonnegative coordinates, then it flows into $\mathbf{E} \cap \mathbf{S}$ through its Southwest boundary (so $\mathbf{v}(\mathbf{x}(t), a) \cdot \mathbf{n}(t) \leq 0$ there), but does not flow out through the other parts of its boundary (since $\mathbf{v}(\mathbf{x}(t), a) \cdot \mathbf{n}(t)=0$ there, by (19)). Thus the derivative in (22) is nonnegative, $\operatorname{so} \operatorname{Prob}(\tilde{\mathbf{x}} \in \mathbf{E} \mid a)$ is nondecreasing in $a$. Similarly, if the coordinates of $\mathbf{v}(\mathbf{x}(t), a)$ are nonincreasing in $a$ then the rate of flow in (22) is nonincreasing in $a$. Thus $\operatorname{Prob}(\tilde{\mathbf{x}} \in \mathbf{E} \mid a)$ is concave in $a$ and $f(\mathbf{x} \mid a)$ satisfies CISP.

Q.E.D.

It is now easy to construct densities $f(\mathbf{x} \mid a)$ which satisfy NISP, CISP and also the MLR property. To do this choose $\mathbf{v}(\mathbf{x}, a)$ as in Lemma 2 so that we also have

$$
f_{a}(x, y \mid a) / f(x, y \mid a)=-\left[u_{x}(x, y, a)+v_{y}(x, y, a)\right] / f(x, y \mid a)
$$

nondecreasing in $x$ and $y$. As an example, let $f(x, y \mid 0)$ be uniform on $\mathbf{S}$, let $u(x, y, a)$ $=\left(x-x^{2}\right)(1-a) \epsilon$, and let $v(x, y, a)=\left(y-y^{2}\right)(1-a) \epsilon$. Then (21) gives

$$
f(x, y \mid a)=1+(x+y-1)\left(2 a-a^{2}\right) \epsilon \text { for }(x, y) \in \mathbf{S} .
$$

If $\epsilon<1$, then this is a strictly positive probability density function on $\mathbf{S}$ for all $a \in[0,1]$, and it satisfies CISP, NISP and the MLR property. Also, this density satisfies neither Jewitt's (1988) independence assumption nor Sinclair-Desgagné's (1994) GCDFC. Clearly it would be easy to generate an enormous range of examples like this. Of course, if one uses affiliation to get NISP, as in the end of Section 6, then MLR follows automatically.

On the other hand, to use this approach to confirm NISP and CISP for a prespecified $f(\mathbf{x} \mid a)$, one must construct an appropriate probability density flow $\mathbf{v}(\mathbf{x}, a)$ corresponding to $f(\mathbf{x} \mid a)$. We show how to do this in the next section. 
Finally, the conditions in Lemma 2, for some given vector flow $\mathbf{v}(\mathbf{x}, a)$, are sufficient for NISP and CISP, but not necessary. However, it seems reasonable to conjecture that, if $f(x, y \mid a)$ satisfies NISP and/or CISP, then there exists a vector flow $\mathbf{v}(\mathbf{x}, a)$ as in Lemma 2. I have not been able to prove this, however.

\section{LOCAL CONDITIONS SUFFICIENT FOR NISP AND CISP}

The previous section reduced NISP and CISP to the existence of certain probability density flows. This allowed us to construct densities satisfying NISP and CISP. However, to check NISP and CISP for a given density, $f(x, y \mid a)$, we must construct vector flows satisfying equations (17) and (19), and then check the conditions in Lemma

2. This is relatively straightforward, and reduces the hard-to-check global NISP and CISP conditions to convenient local conditions.

Let $g(x \mid a)$ and $G(x \mid a)$ be the marginal density and cumulative distribution function of $\tilde{x}$, and let $h(y \mid x, a)$ and $H(y \mid x, a)$ be the conditional density and cumulative distribution function of $\tilde{y}$ given $\tilde{x}=x$. Thus the joint density $f(x, y \mid a)=g(x \mid a) h(y \mid x, a)$. With this notation, the following lemma allows us to check NISP and CISP.

LEMMA 3: Suppose there are functions $\phi(x, y, a)$ and $\psi(x, y, a)$ with

$$
\phi_{x}(x, y, a)=-\psi_{y}(x, y, a)
$$

and

$$
\phi(0, y, a)=\phi(1, y, a)=\psi(x, 0, a)=\psi(x, 1, a)=0
$$

and such that the vector field

$$
\begin{aligned}
& \mathbf{v}(\mathbf{x}, a)=-\left(G_{a}(x \mid a) h(y \mid x, a)+\phi(x, y, a),\right. \\
& \left.\quad g(x \mid a) H_{a}(y \mid x, a)-G_{a}(x \mid a) H_{x}(y \mid x, a)+\psi(x, y, a)\right)
\end{aligned}
$$


has nonnegative coordinates for all $(x, y) \in[0,1] \times[0,1]$. Then the NISP condition holds. Similarly, if there are functions $\phi(x, y, a)$ and $\psi(x, y, a)$ satisfying (23) and (24) and such that the coordinates of (25) are nonincreasing in a, then CISP holds.

ProOF: Using the product rule to calculate $f_{a}(x, y \mid a)$ shows that $\mathbf{v}(\mathbf{x}, a)$ satisfies (17). The coordinates also satisfy (19) since $G_{a}(0 \mid a)=G_{a}(1 \mid a)=H_{a}(0 \mid x, a)=$ $H_{a}(1 \mid x, a)=H_{x}(0 \mid x, a)=H_{x}(1 \mid x, a)=0$. Now apply Lemma $2 . \quad$ Q.E.D.

The density flow in Lemma 3 builds on the basic density flow given by:

$$
\mathbf{v}^{b}(\mathbf{x}, a)=\left(-G_{a}(x \mid a) h(y \mid x, a),-g(x \mid a) H_{a}(y \mid x, a)+G_{a}(x \mid a) H_{x}(y \mid x, a)\right) .
$$

This density flux may seem somewhat ad hoc. To make sense of it, note that this flux can be broken up into three separate parts. First, the flux $-G_{a}(x \mid a) h(y \mid x, a)$, in the first coordinate, ensures that the marginal density $g(x \mid a)$ evolves appropriately as $a$ rises. Dividing this density flux by the density, $g(x \mid a) h(y \mid x, a)$, gives the flow velocity in the $x$ direction, $-G_{a}(x \mid a) / g(x \mid a)$. The specification of the basic density flow in (26) is chosen to make this velocity independent of $y$. Of course, other specifications are possible, and may yield more useful conditions in some applications.

The second coordinate involves two flows. First, the $-g(x \mid a) H_{a}(y \mid x, a)$ term represents a flux in the $y$ direction with corresponding velocity $-H_{a}(y \mid x, a) / h(y \mid x, a)$. This causes the conditional distribution, $h(y \mid x, a)$, to evolve appropriately as $a$ rises. If the two signals, $\tilde{x}$ and $\tilde{y}$ are independent, then these are the only two effects.

However, if $\tilde{x}$ and $\tilde{y}$ are not independent, then there is a third flow, with flux in the $y$ direction given by $G_{a}(x \mid a) H_{x}(y \mid x, a)$. To understand this flow, suppose $G_{a}(x \mid a)<0$, so the first flow above is in the direction of increasing $x$. This first flow will then influence the distribution of $\tilde{y}$ given $\tilde{x}=x$ by mixing the distribution of $\tilde{y}$ given $\tilde{x}=x-d x$ into its distribution given $\tilde{x}=x$, where $d x>0$. The role of the third flow is to counteract this mixing effect. 
Thus, if, e.g., $\tilde{x}$ and $\tilde{y}$ are positively correlated, the mixing effect tends to shift the distribution of $\tilde{y}$ at $\tilde{x}=x$ down, since the distribution of $\tilde{y}$ at $\tilde{x}=x-d x$ is lower than its distribution at $\tilde{x}=x$. However, in this case, $H_{x}(y \mid x, a)$ is negative. This, together with $G_{a}(x \mid a)$ negative, means that the flux, $G_{a}(x \mid a) H_{x}(y \mid x, a)$, in the $y$ direction, is positive. This shifts the distribution of $\tilde{y}$ given $\tilde{x}=x$ back up, as required.

The following propositions give some illustrative applications of Lemma 3.

Proposition 6: Suppose $G(x \mid a)$ is nonincreasing in $a$, and $H(y \mid x, a)$ is nonincreasing in $x$ and $a$. Then NISP holds.

PROOF: Let $\phi \equiv \psi \equiv 0$ in (25). Then since $G(x \mid a)$ is nonincreasing in $a$, the first coordinate of $\mathbf{v}(\mathbf{x}, a)$ is nonnegative. Similarly, the second coordinate is nonnegative since $g(x \mid a) H_{a}(y \mid x, a)$ is nonpositive and $G_{a}(x \mid a) H_{x}(y \mid x, a)$ is nonnegative. Q.E.D.

SECOND PROOF: This result can also be proven without using the vector calculus machinery from the previous section. To do this, let $\mathbf{E}$ be an increasing set, and let $y=b(x)$ be the curve describing the Southwest boundary of $\mathbf{E} \cap \mathbf{S}$. Let $P=\operatorname{Prob}(\tilde{\mathbf{x}} \in$ E) be the probability that the signal $\tilde{\mathbf{x}}$ is in $\mathbf{E}$. Then

$$
P=\int_{x=0}^{1} \int_{y=b(x)}^{1} g(x \mid a) h(y \mid x, a) d y d x=\int_{0}^{1} g(x \mid a)[1-H(b(x) \mid x, a)] d x .
$$

Thus, assuming one can differentiate under the integral sign,

$$
d P / d a=\int_{0}^{1} g_{a}(x \mid a)[1-H(b(x) \mid x, a)] d x-\int_{0}^{1} g(x \mid a) H_{a}(b(x) \mid x, a) d x=I_{1}-I_{2},
$$

where $I_{1}$ and $I_{2}$ are the obvious integrals. Here $I_{2}$ is nonpositive since $H_{a}(b(x) \mid x, a)$ is, so the second term, $-I_{2}$, is nonnegative.

For the first term, integrate $I_{1}$ by parts, yielding

$$
\begin{aligned}
I_{1}=\left.G_{a}(x \mid a)[1-H(b(x) \mid x, a)]\right|_{x=0} ^{1}+\int_{0}^{1} G_{a}(x \mid a) H_{x}(b(x) \mid x, a) d x \\
\quad+\int_{0}^{1} G_{a}(x \mid a) h(b(x) \mid x, a) b^{\prime}(x) d x=0+I_{1 a}+I_{1 b}
\end{aligned}
$$


where the first term is zero since $G_{a}(0 \mid a)=G_{a}(1 \mid a)=0$. Here $I_{1 a}$ is nonnegative since $G_{a}(x \mid a)$ and $H_{x}(b(x) \mid x, a)$ are both nonpositive, and $I_{1 b}$ is nonnegative since $G_{a}(x \mid a)$ and $b^{\prime}(x)$ are both nonpositive.

Q.E.D.

Note that $H(y \mid x, a)$ nonincreasing in $x$ says that, when $\tilde{x}$ increases, the conditional distribution of $\tilde{y}$ is nondecreasing in the sense of first order stochastic dominance. This means that $\tilde{x}$ and $\tilde{y}$ are positively related, but is weaker than affiliation. Similarly, $G(x \mid a)$ and $H(y \mid x, a)$ nonincreasing in a imply that the marginal distribution of $\tilde{x}$, and the conditional distribution of $\tilde{y}$, respectively, are nondecreasing in $a$ in the sense of first order stochastic dominance. These are weaker than the corresponding MLR (and so, affiliation) properties. $^{8}$ Thus, the conditions in Proposition 6 are more general than the affiliation conditions mentioned at the end of Section 6 above. ${ }^{9}$

Proposition 7: Suppose $G_{a}(x \mid a)$ and $H_{a}(y \mid x, a)$ are negative (this follows from strict versions of the corresponding MLR properties for $\tilde{x}$ and $\tilde{y})$. Assume also that $g(x \mid a)$ and $h(y \mid x, a)$ are strictly positive on $[0,1]$. Finally assume $H_{x}(y \mid x, a)<0$ (so

8 To see these results, note that Milgrom and Weber's (1982) Theorem 5 implies that, for $\tilde{x}, \tilde{y}$ and $a$ affiliated, the expectation $E[\Phi(\tilde{x}) \mid a]$ is nondecreasing in $a$, and $E[\Psi(\tilde{y}) \mid \tilde{x}=x, a]$ is nondecreasing in $x$ and $a$, for any nondecreasing functions $\Phi(\cdot)$ and $\Psi(\cdot)$. Taking $\Phi(\cdot)$ to be $\chi_{(x, \infty)}(\cdot)$, the characteristic function of $(x, \infty)$, and $\Psi(\cdot)$ $=\chi_{(y, \infty)}(\cdot)$, shows that affiliation implies the conditions in Proposition 6 .

9 The need for $H(y \mid x, a)$ to be nonincreasing in $x$ also shows why the MLR property was not, by itself, a sufficiently strong monotonicity condition for Sinclair-Desgagné (1994) to use in justifying the first order approach. He therefore required a stronger Assumption, such as (A8), discussed in Section 3 above. Indeed, recognizing that MLR was insufficient by itself for the multi-signal case is one of the important insights in Sinclair-Desgagné (1994). For example, I doubt that I could have obtained the results for a risk averse principal, in Section 6 above, without the benefit of this insight. Proposition 6, however, suggests that one can replace (A8) by MLR and $H(y \mid x, a)$ nonincreasing in $x$, and so, avoid the ancillarity result for Sinclair-Desgagné's approach in Proposition 1 above, even if the principal is risk averse. 
$\tilde{x}$ and $\tilde{y}$ are positively related). Then the conditions

$$
\begin{gathered}
h_{a}(y \mid x, a) / h(y \mid x, a) \leq-G_{a a}(x \mid a) / G_{a}(x \mid a) \\
g_{a}(x \mid a) / g(x \mid a) \leq-H_{a a}(y \mid x, a) / H_{a}(y \mid x, a) \\
H_{a x}(y \mid x, a) / H_{x}(y \mid x, a) \leq-G_{a a}(x \mid a) / G_{a}(x \mid a)
\end{gathered}
$$

are sufficient to ensure CISP.

ProOF: Let $\phi \equiv \psi \equiv 0$ in (25) and consider the first coordinate of $\mathbf{v}(\mathbf{x}, a)$. This is nonincreasing in $a$ if

$$
\partial\left[G_{a}(x \mid a) h(y \mid x, a)\right] / \partial a=G_{a}(x \mid a) h_{a}(y \mid x, a)+G_{a a}(x \mid a) h(y \mid x, a) \geq 0 .
$$

Using $G_{a}(x \mid a)<0, h(y \mid x, a)>0$ and (27) shows (30) holds. Similarly, (28) and (29) show that the second coordinate of $(25)$ is nonincreasing in $a$. Q.E.D.

Just as was the case for Proposition 6, there is a second proof of Proposition 7 which avoids the vector calculus machinery of Section 7 (see Conlon (2008)). However, this alterative proof is as opaque as the alternative proof of Proposition 6 .

Note that, for all $x$, the left hand side of (27) is nonnegative for some values of $y$. Thus, since $G_{a}(x \mid a)<0,(27)$ implies $G_{a a}(x \mid a) \geq 0$ for all $x$, and so, implies the CDFC for $\tilde{x}$. Similarly (28) implies the CDFC for $\tilde{y}$, conditional on $\tilde{x}=x$. Of course, (27) and (28) put additional restrictions on the $\tilde{x}$ and $\tilde{y}$ distributions, beyond the CDFC. This is not surprising since, as Jewitt (1988, p. 1184) points out, the CDFC for each of the random variables $\tilde{x}$ and $\tilde{y}$, separately, is not enough to justify the first-order approach, even if the signals are independent. This is because the product of two concave functions is often not concave (see the discussion of Equation (31) below). 
Condition (29), finally, says that an increase in $a$ should not make $H_{x}(y \mid x, a)$ fall too quickly, i.e., should not increase the correlation between $\tilde{x}$ and $\tilde{y}$ too much. Under independence, $H_{a x}(y \mid x, a)=0$, so (29) follows from $G_{a a}(x \mid a) \geq 0$, and so, from (27).

To illustrate these conditions, assume $\tilde{x}$ and $\tilde{y}$ are independent for specificity, and let them belong to the first class of distributions in LiCalzi and Spaeter (2003), so

$$
G(x \mid a)=x+\beta_{1}(x) \gamma_{1}(a) \text { and } H(y \mid a)=y+\beta_{2}(y) \gamma_{2}(a)
$$

for $x, y \in[0,1]$, where the $\beta_{i}(\cdot)$ are nonnegative and concave on $[0,1]$, with $\beta_{i}(0)$ $=\beta_{i}(1)=0$ and $\left|\beta_{i}^{\prime}(\cdot)\right| \leq 1$, and the $\gamma_{i}(\cdot)$ are decreasing and convex with $\left|\gamma_{i}(\cdot)\right|<1$. Conditions (27) and (28) then impose the additional restrictions

$$
\frac{\beta_{2}^{\prime}(y) \gamma_{2}^{\prime}(a)}{1+\beta_{2}^{\prime}(y) \gamma_{2}(a)} \leq-\frac{\gamma_{1}^{\prime \prime}(a)}{\gamma_{1}^{\prime}(a)} \text { and } \frac{\beta_{1}^{\prime}(x) \gamma_{1}^{\prime}(a)}{1+\beta_{1}^{\prime}(x) \gamma_{1}(a)} \leq-\frac{\gamma_{2}^{\prime \prime}(a)}{\gamma_{2}^{\prime}(a)},
$$

respectively. These are stronger than CDFC in $\tilde{x}$ and $\tilde{y}$ separately, as expected.

The conditions in Proposition 7 are meant to be illustrative, and are by no means canonical. One may nevertheless ask how close these conditions are to best possible, and whether uniformly best, canonical results are possible.

First, the conditions are, in a sense, a point on the Pareto frontier of best possible conditions. To see this suppose there is an increasing set, $\mathbf{E}_{0}$, such that two of the conditions hold as equalities in $\mathbf{E}_{0} \cap \mathbf{S}$, while the third is violated throughout this set. Then CISP is violated for $\mathbf{E}_{0}$. This can be seen since the vector $\mathbf{v}^{b}(\mathbf{x}, a)$ in (26) will have coordinates which are nondecreasing in $a$, with at least one coordinate increasing in $a$. Thus, by the logic of the proof of Lemma $2, \operatorname{Prob}\left(\tilde{\mathbf{x}} \in \mathbf{E}_{0}\right)$ is strictly convex in $a$, violating CISP. In other words, it is not possible to weaken any of the conditions in Proposition 7 without strengthening one of the other conditions.

Second, these conditions can be compared to various necessary conditions for CISP. For example, let $\tilde{x}$ and $\tilde{y}$ be independent for specificity, and consider the increasing set, 
$\mathbf{E}_{\mathbf{x}}=\{\hat{\mathbf{x}}: \hat{\mathbf{x}} \geq \mathbf{x}\}$, for a fixed $\mathbf{x}=(x, y)$. Thus, $\mathbf{E}_{\mathbf{x}} \cap \mathbf{S}$ is a rectangle with lower left hand corner $\mathbf{x}$. CISP then requires, not just that $(1-G(x \mid a))$ and $(1-H(y \mid a))$ be concave in $a$ separately, but that the product, $\operatorname{Prob}\left(\tilde{\mathbf{x}} \in \mathbf{E}_{\mathbf{x}}\right)=(1-G(x \mid a))(1-H(y \mid a))$, be concave in $a$ as well. This in turn requires that

$$
\begin{aligned}
-d^{2} \operatorname{Prob}(\tilde{\mathbf{x}} \in & \left.\mathbf{E}_{\mathbf{x}}\right) / d a^{2}=\left[(1-H(y \mid a)) G_{a a}(x \mid a)-G_{a}(x \mid a) H_{a}(y \mid a)\right] \\
& +\left[(1-G(x \mid a)) H_{a a}(y \mid a)-G_{a}(x \mid a) H_{a}(y \mid a)\right]=T_{1}+T_{2} \geq 0,
\end{aligned}
$$

where $T_{1}$ and $T_{2}$ represent the first and second bracketed terms, respectively.

Now, in the independence case, we can ignore (29) in Proposition 7. Also (allowing for independence), sufficient conditions (27) and (28) were derived from essentially

$$
G_{a a}(x \mid a) h(y \mid a)+G_{a}(x \mid a) h_{a}(y \mid a) \geq 0 \text { and } H_{a a}(y \mid a) g(x \mid a)+H_{a}(y \mid a) g_{a}(x \mid a) \geq 0
$$

respectively. Integrating the left hand side of the first inequality in (32) gives

$$
\begin{aligned}
& \int_{y}^{1}\left[G_{a a}(x \mid a) h(\hat{y} \mid a)+G_{a}(x \mid a) h_{a}(\hat{y} \mid a)\right] d \hat{y} \\
& \quad=(1-H(y \mid a)) G_{a a}(x \mid a)-G_{a}(x \mid a) H_{a}(y \mid a)=T_{1},
\end{aligned}
$$

and similarly for $T_{2}$. Thus, for $T_{1} \geq 0$ and $T_{2} \geq 0$, the inequalities in (32) must hold on average. The sufficient conditions in (32) are then more restrictive than the necessary condition in (31) in exactly two ways: (a) they impose their constraints pointwise, rather than on average, and (b) they require $T_{1} \geq 0$ and $T_{2} \geq 0$ separately, rather than just $T_{1}+T_{2} \geq 0$. Conditions (27) and (28) are then essentially convenient rearrangements of those in (32).

Of course, it is in the nature of easy-to-check local conditions that they should be imposed pointwise, so drawback (a) is inevitable in this type of result. On the other 
hand, drawback (b) suggests that (27) can be weakened if we are willing to strengthen (28) and visa versa. This is the role of the $\phi$ and $\psi$ functions in (25), and specific applications will presumably require clever choices of these functions.

For example, the conditions in Proposition 7 do not imply, and are not implied by, the GCDFC, but a careful choice of the $\phi$ and $\psi$ functions in (25) shows that the GCDFC implies CISP. The GCDFC requires either that $g(x \mid a)[1-H(y \mid x, a)]$ be concave in $a$ for all $(x, y) \in[0,1] \times[0,1]$, or that $h(y \mid a)[1-G(x \mid y, a)]$ be concave in $a$ for all $(x, y) \in[0,1] \times[0,1]$ (with $h(y \mid a)$ and $G(x \mid y, a)$ the obvious marginal density and conditional cumulative distribution functions). While these conditions cannot be obtained from Proposition 7, they can be derived from Lemma 3, as shown in the following proposition.

Proposition 8: The GCDFC implies the CISP condition.

ProOF: For specificity, assume $g(x \mid a)[1-H(y \mid x, a)]$ concave in $a$ for all $(x, y) \in$ $[0,1] \times[0,1]$. Apply Lemma 3 with $\phi(x, y, a)=[1-h(y \mid x, a)] G_{a}(x \mid a)$ and $\psi(x, y, a)$ $=G_{a}(x \mid a) H_{x}(y \mid x, a)+[H(y \mid x, a)-y] g_{a}(x \mid a)$. These functions satisfy (23) and (24). In addition, with these functions, the vector field in (25) becomes

$$
\mathbf{v}(\mathbf{x}, a)=-\left(G_{a}(x \mid a), g(x \mid a) H_{a}(y \mid x, a)+[H(y \mid x, a)-y] g_{a}(x \mid a)\right) .
$$

Now, $g(x \mid a)[1-H(y \mid x, a)]$ is concave in $a$ for all $(x, y) \in[0,1] \times[0,1]$, by GCDFC. Plugging $y=0$ into this shows that $g(x \mid a)$ is concave in $a$, so $g_{a a}(x \mid a) \leq 0$ for all $x \in[0,1]$. Integrating gives $0 \geq \int_{0}^{1} g_{a a}(x \mid a) d x=\left(d^{2} / d a^{2}\right) \int_{0}^{1} g(x \mid a) d x=d^{2} 1 / d a^{2}$ $=0$, so $g_{a a}(x \mid a)=0$ almost everywhere (this also follows from Proposition 1 above). Thus, since $G_{a a}(0 \mid a)=0$, it follows that $G_{a a}(x \mid a)=0$ for all $x$, so the first coordinate of $\mathbf{v}(\mathbf{x}, a)$ in (33) is constant in $a$, and so trivially nonincreasing. 
Next, concavity of $g(x \mid a)[1-H(y \mid x, a)]$ in $a$, plus $g_{a a}(x \mid a)=0$, yields

$$
g(x \mid a) H_{a a}(y \mid x, a)+2 g_{a}(x \mid a) H_{a}(y \mid x, a) \geq 0 .
$$

Also, the second coordinate in (33) is nonincreasing in $a$ if

$$
g(x \mid a) H_{a a}(y \mid x, a)+2 g_{a}(x \mid a) H_{a}(y \mid x, a)+[H(y \mid x, a)-y] g_{a a}(x \mid a) \geq 0 .
$$

Now GCDFC implies both $g_{a a}(x \mid a)=0$ and (34), and these in turn imply (35). Both coordinates of (33) are therefore nonincreasing, so Lemma 3 yields CISP. $\quad$ Q.E.D.

Of course, it may be more natural to derive the GCDFC directly, as in SinclairDesgagné (1994). However, Proposition 8 illustrates the flexibility of Lemma 3. This flexibility may be especially useful in applications with asymmetries between signals, such as contingent monitoring problems (e.g., Fagart and Sinclair-Desgagné (2007)).

On the other hand, I do not believe that it is possible to find "canonical" local conditions implying NISP or CISP. Since NISP and CISP are global conditions, it seems doubtful that one can find local conditions which would be both necessary and sufficient for them. For any local conditions, there should exist some function, $F(x, y \mid a)$, which violates these conditions only in some small region, while NISP and/or CISP still hold. Of course, this does not deny that there may well be conditions which, while imperfect, are nevertheless more general than those in Propositions 6 and 7. For an attempt along these lines which, however, I believe is of limited value, see Conlon (2008).

The above can all be extended to the $n$-signal case. For example, consider the three signal case, with density $f(x, y, z \mid a)=g(x \mid a) h(y \mid x, a) k(z \mid x, y, a)$. Then, leaving off the arguments for brevity, (25) should be replaced by the vector field

$$
\begin{aligned}
& \mathbf{v}=-\left(G_{a} h k+\phi^{1}, g H_{a} k-G_{a} H_{x} k+\phi^{2},\right. \\
& \left.\quad g h K_{a}-G_{a} h K_{x}-g H_{a} K_{y}+G_{a} H_{x} K_{y}+\phi^{3}\right),
\end{aligned}
$$


where $G, H$, and $K$ are the obvious cumulative distribution functions, and the functions $\phi^{i}(x, y, z, a)$ satisfy $\phi_{x}^{1}+\phi_{y}^{2}+\phi_{z}^{3}=0$ and the analogue of (24). One can then obtain results like Propositions 6 and 7 above. The approach is therefore quite flexible. ${ }^{10}$

\section{OTHER EXTENSIONS}

The above method allows other extensions. The basic approach is to find conditions which imply that the function $u\left(s^{*}(\mathbf{x})\right)$ is in some restricted class, and then find conditions on the density, $f(\mathbf{x} \mid a)$ such that the mapping, (13), from $\varphi(\mathbf{x})$ to $\varphi^{T}(a)$, maps this restricted class into concave functions (see also Jewitt (1988, p. 1189-90)).

For example, consider a two-signal case, with signals $\tilde{x}$ and $\tilde{y}$. Suppose the likelihood ratio, $f_{a}(x, y \mid a) / f(x, y \mid a)$, is nondecreasing and submodular in $(x, y)$ (so its cross partial is nonpositive), and that $\omega(z)$ in (10) above is nondecreasing concave. Then it can be shown that $\varphi(\mathbf{x}) \equiv u\left(s^{*}(\mathbf{x})\right)$ is also nondecreasing and submodular. Next suppose $\operatorname{Prob}\left(\tilde{x} \geq x_{0}\right.$ or $\left.\tilde{y} \geq y_{0} \mid a\right)$, is concave in $a$ for all $\left(x_{0}, y_{0}\right)$. Then it can be shown that the agent's payoff, $U\left(s^{*}(\cdot), a\right)=\varphi^{T}(a)-a$, is concave, and the first-order approach is valid (this result was suggested to me by Ian Jewitt; for details see Conlon (2008)). This generalizes Jewitt's other multi-signal result, i.e. his Theorem

2. However, it is not clear how to extend this beyond the two-signal case.

Next (as Ian Jewitt has also pointed out to me), if one is willing to assume that

10 One can also use the vector flows from Section 7 to generate state space representations of the technology. Specifically, divide the flux $\mathbf{v}(\mathbf{x}, a)$ by the density $f(\mathbf{x} \mid a)$ to get the flow velocity, $[1 / f(\mathbf{x} \mid a)] \mathbf{v}(\mathbf{x}, a)$. Suppose the differential system $\partial \mathbf{x} / \partial a=$ $[1 / f(\mathbf{x} \mid a)] \mathbf{v}(\mathbf{x}, a)$ has a unique solution, $\mathbf{x}\left(a, \mathbf{x}_{0}\right)$, for each initial point $\mathbf{x}_{0}$ in the support of $f(\mathbf{x} \mid a=0)$. Then the set of these initial points $\mathbf{x}_{0}$ can be treated as the state space, with density $p\left(\mathbf{x}_{0}\right)=f\left(\mathbf{x}_{0} \mid a=0\right)$. If this construction is applied to $\mathbf{v}^{b}(\mathbf{x}, a)$ in (26), one gets roughly the state space representation in Proposition 3 above (see Conlon (2008)). This approach may also help in obtaining other state space representations. 
the $n-1$ signals in $\tilde{\mathbf{x}}_{-h}$ are ancillary (see Proposition 1 above), then one can extend the Sinclair-Desgagné (1994) results by imposing conditions on the remaining dimension $h$. For example, using the Rogerson (1985) approach leads to the original SinclairDesgagné conditions. The Jewitt (1988) approach could also be used here. It would also be interesting to extend the approach in Brown et al. (1986), building on increasing marginal cost of effort and nonseparabilities between effort and income.

Finally, Ian Jewitt also suggests a third result: Assume $f(\mathbf{x} \mid a)$ is affiliated (i.e., $\left.M T P_{2}\right)$ in $\mathbf{x}$, and let $f_{a a}(\mathbf{x} \mid a) / f(\mathbf{x} \mid a)$ be nonincreasing in $\mathbf{x}$. Then for any nondecreasing function $\varphi(\mathbf{x}), \varphi^{T}(a)=\int \varphi(\mathbf{x}) f(\mathbf{x} \mid a) d \mathbf{x}$ is concave in $a$. To see this, use

$$
\int\left[f_{a a}(\mathbf{x} \mid a) / f(\mathbf{x} \mid a)\right] f(\mathbf{x} \mid a) d \mathbf{x}=\left(d^{2} / d a^{2}\right) \int f(\mathbf{x} \mid a) d \mathbf{x}=0,
$$

so

$$
\frac{d^{2}}{d a^{2}} \int \varphi(\mathbf{x}) f(\mathbf{x} \mid a) d \mathbf{x}=\int \varphi(\mathbf{x}) \frac{f_{a a}(\mathbf{x} \mid a)}{f(\mathbf{x} \mid a)} f(\mathbf{x} \mid a) d \mathbf{x}=-\operatorname{Cov}\left(\varphi(\tilde{\mathbf{x}}),-\frac{f_{a a}(\tilde{\mathbf{x}} \mid a)}{f(\tilde{\mathbf{x}} \mid a)}\right) .
$$

This is nonpositive, since the covariance of the two nondecreasing functions, $\varphi(\mathbf{x})$ and $-f_{a a}(\mathbf{x} \mid a) / f(\mathbf{x} \mid a)$, of the affiliated vector $\tilde{\mathbf{x}}$, is nonnegative. Thus $\varphi^{T}(a)$ is concave. By Lemma 1, this implies CISP.

\section{CONCLUSION: COMPARISON OF THE TWO APPROACHES}

This paper has derived new multi-signal extensions of the two major sets of conditions justifying the first-order approach to moral hazard principal-agent problems. In the past, one of these sets of conditions, the one developed by Rogerson (1985), seems to have been considerably more popular than the other set, developed by Jewitt (1988).

However, the Jewitt approach may be more general in some ways. For example, suppose the principal sees a number of signals, $\tilde{x}_{1}, \tilde{x}_{2}, \ldots, \tilde{x}_{n}$, and suppose that the signals are independent. If the Jewitt conditions in Proposition 2 hold for each signal 
separately, then they clearly also hold for the vector of signals. However, if the $C D F C$ holds for each signal separately, CISP need not hold, as shown in Section 8 above.

Furthermore, suppose the signals $\tilde{x}_{1}, \tilde{x}_{2}, \ldots, \tilde{x}_{n}$ are iid. Then CISP must break down as the number of signals $n \rightarrow \infty$. For if $F(x \mid a)$ is the common cumulative distribution function of the $\tilde{x}_{i}$, then the probability of the increasing set $\mathbf{E}_{\hat{x}}=\{\mathbf{x}$ : $x_{i} \geq \hat{x}$ for all $\left.i\right\}$ is $\operatorname{Prob}\left(\tilde{\mathbf{x}} \in \mathbf{E}_{\hat{x}}\right)=[1-F(\hat{x} \mid a)]^{n}$. This ceases to be concave in $a$ as $n \rightarrow \infty$, so CISP breaks down.

This is related to a more general point. Even in the one-signal case, the CDFC must fail if the signal $\tilde{x}$ is sufficiently accurate. For example, let $\tilde{x}$ have mean $\chi(a)$ for some increasing function $\chi(\cdot)$. If $\chi(\cdot)$ is concave, one would expect to find the sort of decreasing returns that CDFC is intended to capture. However, suppose $\tilde{x}$ has a standard deviation much less than some small $\epsilon$. Then $F(x \mid a)$ will be near one for $a<\chi^{-1}(x)-\epsilon$, then fall rapidly for $a$ near $\chi^{-1}(x)$, and be near zero for $a>\chi^{-1}(x)+\epsilon$. This is a reverse s-shaped function in $a$, and so, cannot be convex in $a$, even if the function $\chi(\cdot)$ is extremely concave. ${ }^{11}$

Now, in the iid case considered just above, the vector of signals, $\tilde{\mathbf{x}}$, becomes almost perfectly informative about $a$ as $n \rightarrow \infty$. Thus, since the CDFC fails if signals approach

11 This also explains a seeming contradiction in the contingent monitoring literature, between Proposition 1 in Fagart and Sinclair-Desgagné (2007), and Theorem 1(a) in Dye (1986). Fagart and Sinclair-Desgagné assume that the first order approach is valid, and show that an optimal contingent monitoring policy is "upper tailed" if Jewitt's function, $\omega(\cdot)$ in $(10)$ above $\left(\Delta^{*^{\prime}}(\cdot)\right.$ in Fagart and Sinclair-Desgagné's notation), is convex. Dye, by contrast, without assuming the first order approach, shows that the optimal contingent monitoring policy is always lower tailed if the monitoring technology is sufficiently accurate, regardless of $\omega(\cdot)$. The resolution of this contradiction is that, if the monitoring technology is very accurate, then the CDFC is violated. Thus we must resort to the Jewitt conditions to justify the first order approach in Fagart and Sinclair-Desgagné (2007), and these conditions require $\omega(\cdot)$ to be concave. 
perfect accuracy, it is not surprising that CISP also fails in this case. ${ }^{12}$

In most actual applications, of course, the vector of signals does not approach perfect accuracy, so the breakdown of CISP is not inevitable. However, the above suggest that alternative conditions, such as Jewitt's results and their generalization in Section 4 , are of significant practical use. ${ }^{13}$

Unfortunately, the Jewitt conditions are tied to the concavity, not only of the technology, but also of the payment schedule $s^{*}(\cdot)$. Thus, there are interesting cases where the Jewitt conditions should fail, since the payment schedule $s^{*}(\cdot)$ is often not concave. E.g., managers often receive stock options, face liquidity constraints, or are subject to contingent monitoring, and these can introduce nonconcavities into their objective functions.

However, I believe that it is natural to expect the first order approach itself to fail in such cases, since the agent's overall objective function will tend to be nonconcave if $s^{*}(\cdot)$ is not concave. The fact that the CDFC and CISP imply concavity of the agent's payoff, regardless of the curvature of $s^{*}(\cdot)$, then suggests that the CDFC/CISP conditions are very restrictive, not that the first order approach is widely applicable.

Nevertheless, if researchers want to ignore such problems, and use the first-order approach even when the payment schedule may not be concave, then the generalizations of the CDFC approach, from Sections 5 and 6 above, can be used.

12 Note, in particular, that this breakdown does not imply that the CISP is a poor generalization of the CDFC. As Lemma 1 shows, CISP is necessary and sufficient for $u\left(s^{*}(\mathbf{x})\right)$ nondecreasing in $\mathbf{x}$ to imply $U\left(s^{*}(\cdot), a\right)$ concave in $a$. Thus, given the general Rogerson approach, CISP is best possible.

13 In addition, as Jewitt (1988) points out, the CDFC, and so, its multi-signal generalizations such as CISP, are not satisfied by most standard statistical distributions. 
Dept. of Economics, University of Mississippi, University, MS 38677, U.S.A., jrconlon@olemiss.edu

\section{APPENDIX: PROOF OF LEMMA 1}

First, (14) clearly implies CISP. For $h(\mathbf{x} ; \mathbf{E})$ is nondecreasing in $\mathbf{x}$ when $\mathbf{E}$ is an increasing set, so by (14), $\operatorname{Prob}(\tilde{\mathbf{x}} \in \mathbf{E} \mid a)=h^{T}(a ; \mathbf{E})$ is concave in $a$.

Thus, assume CISP and let $\varphi(\cdot)$ be nondecreasing. For $\alpha<\beta$ let

$$
\varphi_{[\alpha, \beta]}(\mathbf{x})=\max (\alpha, \min (\beta, \varphi(\mathbf{x})))
$$

This equals $\varphi(\mathbf{x})$ when $\alpha \leq \varphi(\mathbf{x}) \leq \beta$, but equals $\alpha$ when $\varphi(\mathbf{x})<\alpha$ and equals $\beta$ when $\varphi(\mathbf{x})>\beta$. Now $\varphi_{[\alpha, \beta]}(\cdot)$ can be approximated uniformly by the sum

$$
\varphi_{[\alpha, \beta]}^{N}(\mathbf{x})=\alpha+\sum_{i=1}^{N}[(\beta-\alpha) / N] h\left(\mathbf{x} ; \mathbf{E}_{i}\right),
$$

where the sets

$$
\mathbf{E}_{i}=\{\mathbf{x}: \varphi(\mathbf{x}) \geq \alpha+(\beta-\alpha)(i / N)\}
$$

are increasing sets (note that (A1) essentially expresses the convex cone of increasing functions in terms of its extreme rays). In addition, the transformation in (13) is linear and also continuous under the uniform norm. Thus, applying this transformation to (A1) indicates that $\varphi_{[\alpha, \beta]}^{T}(a)$ is approximated uniformly by

$$
\left(\varphi_{[\alpha, \beta]}^{N}\right)^{T}(a)=\alpha+\sum_{i=1}^{N}[(\beta-\alpha) / N] h^{T}\left(a ; \mathbf{E}_{i}\right) .
$$

Here $h^{T}\left(a ; \mathbf{E}_{i}\right)=\int h\left(\mathbf{x} ; \mathbf{E}_{i}\right) f(\mathbf{x} \mid a) d \mathbf{x}=\operatorname{Prob}\left(\tilde{\mathbf{x}} \in \mathbf{E}_{i} \mid a\right)$ is concave in $a$ by CISP, so $\left(\varphi_{[\alpha, \beta]}^{N}\right)^{T}(a)$ is concave in $a$ for all $N$. Thus $\varphi_{[\alpha, \beta]}^{T}(a)$ must also be concave in $a$. Next, letting $\alpha \rightarrow-\infty$ and using the monotone convergence theorem shows that $\varphi_{(-\infty, \beta]}^{T}(a)$ is concave in $a$. Similarly, $\varphi_{(-\infty, \infty)}^{T}(a)=\varphi^{T}(a)$ is concave in $a$ Q.E.D. 
REFERENCES

Brown, M., S. H. Chiang, S. Ghosh, And E. Wolfstetter (1986): “A New Class of Sufficient Conditions for the First-Order Approach to the Principal-Agent Problem," Economics Letters, 21, 1-6.

Conlon, J. R. (2008): "Supplement to 'Two New Conditions Supporting the First-Order Approach to Multi-Signal Principal-Agent Problems'," Econometrica Supplementary Material.

Dye, R. A. (1986): "Optimal Monitoring Policies in Agencies," Rand Journal of Economics, 17, 339-350.

FAgart, M.-C. AND B. Sinclair-Desgagné (2007): "Ranking Contingent Monitoring Systems," Management Science, 53, 1501-1509.

Grossman, S. J. And O. D. Hart (1983): "An Analysis of the Principal-Agent Problem," Econometrica, 51, 7-45.

Guesnerie, R., AND J.-J. LAfFont (1978): "Taxing Price Makers," Journal of Economic Theory, 19, 423-455.

Holmström, B. (1979): "Moral Hazard and Observability," Bell Journal of Economics, 10, 74-91.

— (1982): "Moral Hazard in Teams," Bell Journal of Economics, 13, 324340.

JEWITT, I. (1988): "Justifying the First-Order Approach to Principal-Agent Problems," Econometrica, 56, 1177-1190.

_ (2007): "Information Order in Decision and Agency Problems," working paper, Nuffield College, Oxford.

Jewitt, I., O. Kadan, And J. M. Swinkels (2008): "Moral Hazard with Bounded Payments," to appear, Journal of Economic Theory.

LANG, S. (1987): Calculus of Several Variables, Third Edition. New York, N.Y.: Springer-Verlag. 
Lehmann, E. L. (1983): Theory of Point Estimation. New York, N.Y.: Wiley \& Sons.

LiCalzi, M., AND S. Spaeter (2003): "Distributions for the First-Order Approach to Principal-Agent Problems," Economic Theory, 21, 167-173.

Milgrom, P. R., And R. J. Weber (1982): "A Theory of Auctions and Competitive Bidding," Econometrica, 50, 1089-1122.

MirRlEES, J. A. (1999): "The Theory of Moral Hazard and Unobservable Behavior: Part I," Review of Economic Studies, 66, 3-21.

MookherJee, D. (1984): "Optimal Incentive Schemes with Many Agents," Review of Economic Studies, 51, 433-446.

Müller, A., AND D. Stoyan (2002): Comparison Methods for Stochastic Models and Risks. West Sussex, England: John Wiley \& Sons, Ltd.

NAChBin, L. (1965): Topology and Order. Princeton, N.J.: Van Nostrand.

Rogerson, W. P. (1985): "The First-Order Approach to Principal-Agent Problems," Econometrica, 53, 1357-1367.

Ross, S. A. (1973): "The Economic Theory of Agency: The Principal's Problem," American Economic Review, 63, 134-139.

Shavell, S. (1979): "Risk Sharing and Incentives in the Principal and Agent Relationship," Bell Journal of Economics, 10, 55-73.

Sinclair-Desgagné, B. (1994): "The First-Order Approach to Multi-Signal Principal-Agent Problems," Econometrica, 62, 459-465.

_ (2008): "Ancillary Statistics in Principal-Agent Models," working paper, HEC Montréal.

Spence, M., AND R. ZeCKhauser (1971): "Insurance, Information, and Individual Action," American Economic Review, 61, 380-387.

Taylor, A. E., AND W. R. Mann (1983): Advanced Calculus, Third Edition. New York, N.Y.: John Wiley \& Sons, Inc. 